\title{
Nanoscale self-organisation in Mott insulators: a richness in disguise
}

\section{Andrea Ronchi}

Università Cattolica del Sacro Cuore https://orcid.org/0000-0001-8960-4561

\section{Paolo Franceschini}

Università Cattolica del Sacro Cuore

\section{Andrea De Poli}

Università Cattolica del Sacro Cuore

\section{Pia Homm}

KU Leuven

\section{Ann Fitzpatrick}

Diamond Light Source

\section{Francesco Maccherozzi}

Diamond Light Source, Chilton, Didcot, Oxfordshire, OX11 ODE, UK.

\section{Gabriele Ferrini}

Università Cattolica del Sacro Cuore https://orcid.org/0000-0002-5062-9099

\section{Francesco Banfi}

Universite de Lyon, Université Lyon 1 and CNRS https://orcid.org/0000-0002-7465-8417

\section{Sarnjeet Dhesi}

Diamond Light Source https://orcid.org/0000-0003-4966-0002

\section{Mariela Menghini}

IMDEA Nanoscience https://orcid.org/0000-0002-1744-798X

\section{Michele Fabrizio}

International School for Advanced Studies (SISSA) https://orcid.org/0000-0002-2943-3278

\section{Jean-Pierre Locquet}

KU Leuven

Claudio Giannetti ( $\nabla$ claudio.giannetti@unicatt.it )

Università Cattolica del Sacro Cuore https://orcid.org/0000-0003-2664-9492

\section{Article}

Keywords: mott transitions, ultrafast light excitation, nanoscale self ogranization

Posted Date: September 10th, 2021 
DOI: https://doi.org/10.21203/rs.3.rs-852103/v1

License: (c) (1) This work is licensed under a Creative Commons Attribution 4.0 International License. Read Full License 


\section{Nanoscale self-organisation in Mott insulators: a richness in disguise}

3 Andrea Ronchi ${ }^{1,2,3, \dagger}$, Paolo Franceschini ${ }^{1,2,3}$, Andrea De Poli ${ }^{1,3,4}$, Pía Homm ${ }^{2}$, Ann Fitzpatrick ${ }^{5}$,

4 Francesco Maccherozzi $^{5}$, Gabriele Ferrini ${ }^{1,3}$, Francesco Banfi ${ }^{6}$, Sarnjeet S. Dhesi ${ }^{5}$, Mariela Menghini ${ }^{2,7}$,

5 Michele Fabrizio $^{4, \star}$, Jean-Pierre Locquet $^{2}$ \& Claudio Giannetti ${ }^{1,3, \ddagger}$

$6{ }^{1}$ Department of Physics, Università Cattolica del Sacro Cuore, Brescia I-25121, Italy

$7{ }^{2}$ Department of Physics and Astronomy, KU Leuven, Celestijnenlaan 200D, 3001 Leuven, Bel-

8 gium

$9{ }^{3}$ ILAMP (Interdisciplinary Laboratories for Advanced Materials Physics), Università Cattolica del

$17 \dagger$ andrea.ronchi@unicatt.it

18

${ }_{19} \ddagger$ claudio.giannetti@unicatt.it

Italy

^fabrizio@sissa.it
Sacro Cuore, Brescia I-25121, Italy

${ }^{4}$ Scuola Internazionale Superiore di Studi Avanzati (SISSA), Via Bonomea 265, 34136 Trieste,

${ }^{5}$ Diamond Light Source, Didcot, Oxfordshire, OX11 ODE, UK

${ }^{6}$ FemtoNanoOptics group, Université de Lyon, CNRS, Université Claude Bernard Lyon 1, Institut

Lumière Matière, F-69622 Villeurbanne, France

${ }^{7}$ IMDEA Nanociencia, Cantoblanco, 28049, Madrid Spain 
Mott transitions in real materials are first order and almost always associated with lattice distortions, both features promoting the emergence of nanotextured phases. This nanoscale self-organization creates spatially inhomogeneous regions, which can host and protect transient non-thermal electronic and lattice states triggered by light excitation. However, to gain full control of the Mott transition for potential applications in the field of ultrafast switching and neuromorphic computing it is necessary to develop novel spatial and temporal multiscale experimental probes as well as theoretical approaches able to distill the complex microscopic physics into a coarse-grained modelling.

Here, we combine time-resolved X-ray microscopy, which snaps phase transformations on picosecond timescales with nanometric resolution, with a Landau-Ginzburg functional approach for calculating the strain and electronic real-space configurations. We investigate $\mathrm{V}_{2} \mathrm{O}_{3}$, the archetypal Mott insulator in which nanoscale self-organization already exists in the low-temperature monoclinic phase and strongly affects the transition towards the hightemperature corundum metallic phase. Our joint experimental-theoretical approach uncovers a remarkable out-of-equilibrium phenomenon: the photoinduced stabilisation of the long sought monoclinic metal phase, which is absent at equilibrium and in homogeneous materials, but emerges as a metastable state solely when light excitation is combined with the underlying nanotexture of the monoclinic lattice. Our results provide full comprehension of the nanotexture dynamics across the insulator-to-metal transition, which can be readily extended to many families of Mott insulating materials. The combination of ultrafast light excitation and spatial nanotexture turns out to be key to develop novel control protocols in 
correlated quantum materials.

\section{Introduction}

Since its original proposal back in $1949^{1}$, the Mott metal-insulator transition keeps attracting interest. For long, experimentalists and theorists have put lot of effort to understand the microscopic bases of such transition in real materials and model Hamiltonians. However, it has recently become urgent to extend that effort towards understanding the dynamics of the Mott transition on multiple timescales and at length scales much longer than the inter-atomic distances ${ }^{2}$, which are most relevant for potential applications. Indeed, Mott transitions in real materials have a first order character, often very pronounced, so that driving across such transitions requires one phase, formerly metastable, to nucleate, grow, and finally prevail over the other, formerly stable. In addition, real Mott transitions are nearly all the times accompanied by a lattice distortion, which, besides enhancing the first order character of the transition, also constrains the nucleation and growth dynamics, fostering the emergence of nanotextures within the insulator-metal coexistence region ${ }^{3,4}$. Moreover, it may happen that also the lower symmetry crystal structure, usually the insulating phase, is inhomogeneous because of coexisting twins ${ }^{5}$. This is often the case when the elastic strain is directly involved in the structural transition.

Such circumstances might, at first sight, be regarded just as unwanted side effects that mess up the Mott transition. In Mott materials, the onset of high-temperature metallicity is accompanied by the melting of the low-temperature lattice configuration, which is a slow process and implies a complex real-space rearrangement of domains at the nanoscale. This slow dynamics constitutes 
the bottleneck for the realization of electronic volatile switches operating at frequencies as high as several $\mathrm{THz}^{24}$, which often discouraged potential applications of insulator-to-metal transitions (IMT) in real materials either driven by temperature changes or by non-equilibrium protocols such as light excitation. In this framework, specific efforts have been recently devoted to investigate possible transient non-thermal states in vanadium oxides, which undergo temperature-driven IMT of great interest for resistive switching and neuromorphic computing applications ${ }^{6-23}$. Much activity has focused on developing strategies to decouple the electronic and structural changes, with the ultimate goal of achieving all-electronic switching for ultrafast Mottronics. The recent claim of a photoinduced metallic phase of monoclinic $\mathrm{VO}_{2}{ }^{25,26}$ has triggered a huge effort to address to what extent the photoinduced transition is similar to the thermally driven one and whether the electronic and lattice degrees of freedom remain coupled at the nanoscale during and after the light excitation $^{3,27-30}$.

The goal of this work is to finally clarify the role of spatial nanotexture in controlling the Mott transition dynamics and in favouring the decoupling of the electronic and lattice transformations when the system is driven out-of-equilibrium by light pulses. We focus on the archetypal Mott insulator $\mathrm{V}_{2} \mathrm{O}_{3}{ }^{31-34}$, which indeed realises all at once the full complex phenomenology we previously outlined, and thus is the privileged playground to attempt such an effort. A nanotextured metal-insulator coexistence across the equilibrium first order transition in thin films has in fact been observed by near-field infrared microscopy in Ref. 35. Specifically, the metal-insulator coexistence is characterised by a rather regular array of striped metallic and insulating domains oriented along two of the three possible hexagonal axes of the high temperature rhombohedral 
structure, the missing twin possibly being a consequence of the R-plane orientation of the film ${ }^{36}$. Later, it has been observed, still on thin films but now with a $c$-plane orientation and using X-ray Photoemission Electron Microscopy (PEEM) ${ }^{37}$, that the monoclinic insulator is itself nanotextured. In particular, such phase looks like a patchwork of the three equivalent monoclinic twins oriented along the three hexagonal axes of the parent rhombohedral phase. Upon raising the temperature of the monoclinic insulator, metallic domains start nucleating along the interfaces between the monoclinic twins ${ }^{37}$, thus forming stripes coexisting with insulating ones, all of them again oriented along the hexagonal axes, in agreement with the experiment in Ref. 35. The origin of this complex nanoscale self-organization, which was tentatively attributed to the long-range Coulomb repulsion ${ }^{35}$, still remains unexplained.

In this work we develop a coarse-grained approach that is able to capture the real-space lattice and electron dynamics of the IMT in $\mathrm{V}_{2} \mathrm{O}_{3}$. Our model demonstrates that the intrinsic nanotexture is driven by the elastic strain associated to the monoclinic lattice distortion. The full understanding of the transition dynamics also discloses the possibility of stabilizing a non-thermal metallic electronic state, which retains the insulating monoclinic lattice structure. This state is unfavorable at equilibrium and in homogeneous materials, but it can be photoinduced when the electronic population within the vanadium $3 d$ bands is modified by ultrafast light pulses. The intrinsic nanotexture is key to create the strain conditions at the boundaries of the monoclinic twins, which protect and stabilize the non-thermal monoclinic metallic phase. We experimentally demonstrate the existence of such a metastable phase by performing novel synchrotron-based time-resolved X-ray PEEM (trPEEM) experiments with $30 \mathrm{~nm}$ and $80 \mathrm{ps}$ spatial and temporal resolution. The excitation of $\mathrm{V}_{2} \mathrm{O}_{3}$ 
thin films with intense infrared $(1.5 \mathrm{eV})$ ultrashort light pulses turns the material into a metal with the same shear strain nanotexture of the insulating phase. Even though all experimental and theoretical results we are going to present refer to vanadium sesquioxide, they reveal an unexpected richness that may as well emerge in other Mott insulating materials. The role played by the spontaneous nanoscale lattice architectures characterizing first-order IMT provides a new parameter to achieve full control of the electronic phase transformation in Mott materials.

The work is organized as follows. In Sec. 2 we provide an overview of the lattice and electronic transformations which characterize the phase diagram of $\mathrm{V}_{2} \mathrm{O}_{3}$, as well as characterization of the spontaneous nanotexture of the monoclinic insulating phase. This information is crucial since it provides the microscopic bases of the multiscale model of the lattice and electronic transition. In Sec. 3 we introduce the model, based on proper Landau-Ginzburg functionals, and we show how it captures the nanotexture formation as well as the dynamics of the temperature induced phase transition. In Sec. 4 we present the central non-equilibrium results. The multiscale model is extended to treat the non-equilibrium case. The model shows that the nanotexture can favour and stabilize a non-thermal electronic metallic phase which retains the monoclinic shear strain of the low-temperature lattice. We experimentally demonstrate this phenomenon by performing tr-PEEM measurements on $\mathrm{V}_{2} \mathrm{O}_{3}$ thin films disclosing the ultrafast dynamics with spatial resolution. Finally, we also show the possibility of controlling the non-thermal transition dynamics by interface strain engineering. 


\section{Electronic and structural transition in $\mathrm{V}_{2} \mathrm{O}_{3}$ : a brief overview}

The phase diagram and lattice transformation The phase diagram of $\left(\mathrm{V}_{1-x} \mathrm{M}_{x}\right)_{2} \mathrm{O}_{3}, \mathrm{M}=\mathrm{Cr}, \mathrm{Ti}$, is shown in Fig. 1. It includes rhombohedral paramagnetic insulator and metal phases with corundum structure, and a low temperature dome where the system is a monoclinic antiferromagnetic Mott insulator. The effect of pressure is, as expected, to favour the metal phase, alike that of $\mathrm{Ti}$ doping, although, above $32.5 \mathrm{GPa}$, such metal appears to be also monoclinic at room temperature ${ }^{38,39}$. The low-pressure transition from the high temperature corundum structure to the low temperature monoclinic one has a first order nature that weakens with $\mathrm{Cr}$ doping $x$, and maybe turns continuous above $x \simeq 0.03$. For pure $\mathrm{V}_{2} \mathrm{O}_{3}$, the case of interest here (dashed vertical line in Fig. 1) the electronic, magnetic and structural transition that occurs at $T_{c} \simeq 170 \mathrm{~K}$ in bulk crystals has a very pronounced first order character: the jump in resistivity covers almost six orders of magnitude ${ }^{32}$, and the strain-driven rhombohedral-monoclinic martensitic transformation ${ }^{40}$ can be rather destructive if the sample is not dealt with care.

In the rhombohedral phase above $T_{c}, \mathrm{~V}_{2} \mathrm{O}_{3}$ crystallises in a corundum structure, space group $R \overline{3} c$ No. 167. The non-primitive hexagonal unit cell contains six formula units, and has the lattice vectors shown in Fig. 2, where $32,41,42$

$$
a_{H}=b_{H} \simeq 4.936 \AA, \quad c_{H} \simeq 14.021 \AA
$$

and, by convention, we choose $\boldsymbol{a}_{H} \| \mathrm{x}$ and $\boldsymbol{c}_{H} \| \boldsymbol{z}$. The vanadium atoms form honeycomb planes with ABC stacking, see also the Supplementary Material ${ }^{43}$. It follows that each Vanadium has only one nearest neighbour along the hexagonal $\boldsymbol{c}_{H}$-axis. We hereafter denote such vertical pairs as 
'dimers'. Moreover, the two inequivalent $\mathrm{V}$ atoms within each honeycomb plane do not lie on such plane, see Fig. 2: the atoms that form dimers with the plane above/below are shifted down/up. The dimer bond length $d_{0}$ is slightly shorter than the distance $\ell_{0}$ between nearest neighbour $\mathrm{V}$ atoms within the hexagonal planes, specifically

$$
d_{0} \simeq 2.7 \AA, \quad \ell_{0} \simeq 2.873 \AA .
$$

Such difference reflects a trigonal distortion of the oxygen octahedra surrounding each vanadium, which is responsible of the $\mathrm{V}-3 d t_{2 g}$ orbital splitting into a lower $e_{g}^{\pi}$ doublet and an upper $a_{1 g}$ singlet, and is believed to play a crucial role in the Mott metal-insulator transition ${ }^{44}$. In Fig. 3 we draw an oversimplified picture of the Mott transition to emphasise the role of the trigonal crystal field splitting.

The magnetic insulator below $T_{c}$ has a monoclinic crystal structure, space group $I 2 / a$, No. 15 . The structural distortion breaks the $C_{3}$ rotation symmetry around the $c_{H}$-axis, and can be viewed ${ }^{41}$ as a rotation of the atoms in a plane perpendicular to one of three hexagonal axes, $\boldsymbol{a}_{H}, \boldsymbol{b}_{H}$ and $-\boldsymbol{a}_{H}-\boldsymbol{b}_{H}$. The three choices correspond to equivalent monoclinic structures, which are distinguishable only in reference to the parent corundum state. We shall here choose for simplicity the $\boldsymbol{a}_{H} \| \boldsymbol{x}$ rotation axis, which becomes the monoclinic primitive lattice vector $\boldsymbol{b}_{m}$, so that the rotation occurs in the $\boldsymbol{y}-\boldsymbol{z}$ plane, where the monoclinic lattice vectors $\boldsymbol{a}_{m}$ and $\boldsymbol{c}_{m}$ lie. Concerning magnetism, each $\boldsymbol{a}_{m}-\boldsymbol{c}_{m}$ plane is ferromagnetic, while adjacent planes are coupled to each other antiferromagnetically, see Fig. 4. In other words, the dimers, which do lie in the $\boldsymbol{a}_{m}-\boldsymbol{c}_{m}$ plane, are ferromagnetic. Similarly, of the three nearest neighbour bonds in the hexagonal plane, the one 
lying in the $\boldsymbol{a}_{m}-\boldsymbol{c}_{m}$ plane of length $\ell_{y}$ is therefore ferromagnetic, while the other two, of length $\ell_{1}$ and $\ell_{2}$, are antiferromagnetic, see Fig. 4.

Given our choice of the monoclinic twinning, the relation between monoclinic and hexagonal lattice vectors ${ }^{32,41}$ are graphically shown in Fig. 4. However, for later convenience, we hereafter prefer to use a non-primitive pseudo-hexagonal unit cell, see Fig. 5. With such choice the monoclinic phase corresponds to

$$
\begin{aligned}
& a_{H} \simeq 5.002 \AA, \quad b_{H} \simeq 4.974 \AA, \quad c_{H} \simeq 13.953 \AA, \\
& \alpha \simeq 91.73^{\circ}, \quad \beta=90^{\circ}, \quad \gamma \simeq 120.18^{\circ},
\end{aligned}
$$

as opposed to the corundum parameters in Eq. (1), with $\alpha=\beta=90^{\circ}$ and $\gamma=120^{\circ}$. The corundum-to-monoclinic transition is therefore accompanied by a volume expansion of $1.4 \%{ }^{32}$, as expected across a metal-insulator transition.

Considering the nearest neighbour $\mathrm{V}-\mathrm{V}$ distances in the hexagonal plane, $\ell_{y}, \ell_{1}$, and $\ell_{2}$, and the dimer length $d$, see Fig. 4 , in the monoclinic phase as compared to the rhombohedral one, see Eq. (2), $\ell_{y}$ grows by $4 \%, d$ by $1.6 \%$, while $\ell_{1}$ and $\ell_{2}$ are almost unchanged, one is $0.38 \%$ shorter and the other $0.14 \%$ longer. In other words, in accordance with the Goodenough-Kanamori-Anderson rules, all ferromagnetic bonds lengthen, the planar one $\ell_{y}$ quite a bit more than the dimer.

Spontaneous nanotexture of the monoclinic insulating phase As described in Ref. 37, equilibrium Photo-Emission Electron Microscopy (PEEM), exploiting X-ray Linear Dichroism (XLD) ${ }^{46}$ as the contrast mechanism, can be used to investigate the dynamics of the rhombohedral to monoclinic transition in real space. In Fig. 6a we report typical spatially-resolved XLD-PEEM images 
taken at $T=100 \mathrm{~K}$, i.e. fully in the monoclinic insulating phase of $\mathrm{V}_{2} \mathrm{O}_{3}$. In this experiment, Linear Horizontal (LH) $s$-polarized X-ray pulses in resonance with the $\mathrm{V} L_{2,3}$-edge $(520 \mathrm{eV})$ impinge with $75^{\circ}$ incident angle on a $40 \mathrm{~nm} \mathrm{~V}_{2} \mathrm{O}_{3}$ film epitaxially grown on a (0001)- $\mathrm{Al}_{2} \mathrm{O}_{3}$ substrate, therefore with the $c$-axis oriented perpendicular to the surface of the film ${ }^{45}$. For each fixed X-rays polarization (see the grey arrows on top of the images), three different striped insulating nanodomains, indicated with red, blue and yellow colours, are clearly visible. The stripe-like domains are oriented along the edges of the vanadium hexagons, with characteristic dimensions of a few micrometers in length and 200-300 nm in width. The observed spontaneous nanotexture of the monoclinic phase demostrates that the minimization of the total elastic energy drives the formation of domains in which the monoclinic distortion takes place along one of the three equivalent directions. As extensively discussed in Ref. 37, when the system is heated up and the coexistence region is entered, rhombohedral metallic droplets start nucleating at the domain boundaries. Upon further heating up, the domains grow until the insulator-to-metal percolative transition takes place at a metallic filling fraction of the order of 0.45 . The relation between the nature of the striped insulating domains and the monoclinic lattice vectors can be further appreciated by rotating the X-ray polarization and plotting the XLD signal in specific regions (pink square, green circle and yellow triangle in the left panel of Fig. 6a) corresponding to the three different domains. As shown in Fig. 6b, the XLD signal of the three different domains is phase-shifted by $60^{\circ}$ and has a $180^{\circ}$ periodicity.

To interpret these observations, in Section S5 of the Supplementary Material ${ }^{43}$ we calculate the polarization dependence of the XLD contrast within the monoclinic phase. In brief, the $a_{1 g}$ 
singlet and the $e_{g}^{\pi}$ doublet transform, respectively, as the one dimensional, $A_{1} \sim z^{2}$, and two dimensional, $E \sim(x, y)$, irreducible representations of $D_{3}$. The monoclinic distortion generates a mixing between $a_{1 g}$ and the combination of the $e_{g}^{\pi}$ that lies on the $\boldsymbol{a}_{m}-\boldsymbol{c}_{m}$ monoclinic plane, i.e., perpendicular to $\boldsymbol{b}_{m}$. Such combination evidently changes among the three equivalent monoclinic twins, and, because of its directionality, it does contribute to the XLD signal, which we find is minimum (maximum) for in-plane components of the field parallel (perpendicular) to the $\boldsymbol{b}_{m}$ axis. Since the latter can be any of the three hexagonal axes, this immediately explains the observation in Fig. 6b, but also allows us to conclude that the interface between two of the three monoclinic twins is perpendicular to the $\boldsymbol{b}_{m}$ axis of the third one.

\section{Multiscale modelling}

The self-organization of the monoclinic insulating phase on length scales of the order of hundreds nanometers calls for a multiscale theory effort which builds on the microscopic parameters governing the transition but goes beyond by describing the formation of domains at length scales much larger than the lattice unit cell. Already Denier and Marezio ${ }^{41}$ emphasised that the main change that occurs across the rhombohedral to monoclinic transition is actually the displacement of the $\mathrm{V}$ atoms of a dimer towards "the adjacent octahedral voids", see also the Supplementary Material ${ }^{43}$. Such displacement results in a $\theta=1.8^{\circ}$ anticlockwise rotation of the dimers around the $\boldsymbol{b}_{m}$ axis, thus the value of $\alpha \simeq 91.73^{\circ}$ and the $c_{H}$ axis compression in Eq. (3). In addition, the dimer elongates by $1.6 \%$. More specifically, the Vanadium displacement, with our choice of monoclinic axis $\boldsymbol{b}_{m} \| \boldsymbol{x}$, has non negligible components along both $\boldsymbol{y}$ and $\boldsymbol{z}$, the former leading to the $1.8^{\circ}$ dimer 
tilting, and the latter mostly responsible of the $1.6 \%$ stretching of the bond. We remark that the tilting alone accounts not only for the variations of $\alpha$ and $c_{H}$, but also for the increase in $\ell_{y}$, which is the most significant change crossing the structural transition, as well as for the dilation along the hexagonal $a_{H}$, cf. Eq. (1) with (3). Therefore, the deformation of the unit cell across the transition is primarily a consequence of the dimer tilting, namely of the Vanadium displacements along $\boldsymbol{y}$.

Hereafter, we thus make the assumption that the $\boldsymbol{y}$ and $\boldsymbol{z}$ components of the Vanadium displacement, or, equivalently, the dimer tilting and its elongation, correspond to different degrees of freedom, by all means coupled to each other ${ }^{47}$, but each playing its distinctive role. The tilting is ultimately responsible of breaking the threefold rotation symmetry around $\boldsymbol{c}_{H}$, and thus of the rhombohedral to monoclinic transition. On the contrary, the V-displacement along $z$ preserves the $R \overline{3} c$ space group, but increases the trigonal distortion and, concurrently, reduces the dimer bonding strength. Both these effects are believed ${ }^{44}$ to drive $\mathrm{V}_{2} \mathrm{O}_{3}$ towards a Mott insulating state, irrespective of the monoclinic distortion. Similarly, the $C_{3}$ symmetry breaking at the structural transition may not necessarily go along with a metal-insulator transition. DFT-GGA electronic structure calculations predict ${ }^{48}$ that the Fermi surface of the corundum metal is unstable towards a monoclinic distortion. Such weak-coupling Fermi surface instability has its strong coupling counterpart. Indeed, assuming a Mott insulating state in which each Vanadium acts like a spin-1, see Fig. 3, it was shown ${ }^{48}$ that $\mathrm{V}_{2} \mathrm{O}_{3}$ realises on each honeycomb plane a highly frustrated Heisenberg model, with comparable nearest and next-nearest neighbour antiferromagnetic exchange constants ${ }^{49-51}$. Such frustration is efficiently resolved by the monoclinic distortion stabilising the stripe phase shown in Fig. 4. This prediction has got further support by recent inelastic neutron scattering data combined 
with DFT calculations ${ }^{52}$. All the above results suggest that the corundum phase of pure $\mathrm{V}_{2} \mathrm{O}_{3}$ is intrinsically unstable, and destined to turn into a monoclinic phase at low temperature. The fact that such transition happens to coincide with a metal to an antiferromagnetic insulator transition indicates just a strong positive interplay between the lattice instability and the electronic correlations $47,48,52$. However, nothing would prevent the structural and the metal-insulator transitions to occur separately. Indeed, chromium doping, see Fig. 1, does drive a metal-insulator transition without an intervening monoclinic distortion. The opposite case of a monoclinic transition not accompanied by a metal-insulator one is still highly debated. Evidences of a monoclinic metal phase have been observed in $\mathrm{V}_{2} \mathrm{O}_{3}$ at high pressure ${ }^{38,39}$. On the contrary, at ambient pressure the possible existence of a monoclinic metal phase remains so far controversial ${ }^{30,35,37,53-58}$.

The rhombohedral to monoclinic martensitic transformation is therefore driven primarily by the dimer tilting, which, macroscopically, corresponds to a lattice strain. We recall that the infinitesimal strain tensor is defined as

$$
\epsilon_{i j}(\mathbf{r})=\frac{1}{2}\left(\frac{\partial u_{i}(\mathbf{r})}{\partial r_{j}}+\frac{\partial u_{j}(\mathbf{r})}{\partial r_{i}}\right), \quad i, j=1,2,3
$$

where $\boldsymbol{u}(\mathbf{r})$ is the displacement field. It is physically more convenient to define new strain variables 
through

$$
\begin{aligned}
\epsilon & =\frac{\epsilon_{11}+\epsilon_{22}}{2}, \quad \epsilon_{3}=\epsilon_{33} \\
\boldsymbol{\epsilon}_{1} & =\left(\begin{array}{c}
\frac{\epsilon_{11}-\epsilon_{22}}{2} \\
\epsilon_{12}
\end{array}\right)=\epsilon_{1}\left(\begin{array}{c}
\cos \phi_{1} \\
\sin \phi_{1}
\end{array}\right) \\
\boldsymbol{\epsilon}_{2} & =\left(\begin{array}{c}
\epsilon_{13} \\
\epsilon_{23}
\end{array}\right)=\epsilon_{2}\left(\begin{array}{c}
\cos \phi_{2} \\
\sin \phi_{2}
\end{array}\right)
\end{aligned}
$$

with $\epsilon_{i}=\left|\epsilon_{i}\right| \geq 0, i=1,2$, which transform under a rotation around $\boldsymbol{z}$ by a generic angle $\phi$ according to

$$
\begin{aligned}
& \epsilon \rightarrow \epsilon, \quad \epsilon_{1} \rightarrow \epsilon_{1}, \quad \epsilon_{2} \rightarrow \epsilon_{2}, \quad \epsilon_{3} \rightarrow \epsilon_{3}, \\
& \phi_{1} \rightarrow \phi_{1}+2 \phi, \quad \phi_{2} \rightarrow \phi_{2}+\phi .
\end{aligned}
$$

The $C_{3}$ symmetry of the rhombohedral structure implies invariance only under rotations by $\phi=$ $\pm 2 \pi / 3$.

The strain components directly related to the dimer tilting are the shear strain ones, $\epsilon_{13}$ and $\epsilon_{23}$, which transform as the components of a planar vector, see Eq. (6). We can therefore identify the vector $\epsilon_{2}$ in (5) with the two-component order parameter of the rhombohedral to monoclinic transition. Specifically, using the structural data of the corundum and monoclinic structures across the transition $32,41,42$, and in our reference frame, the shear strain order parameter $\boldsymbol{\epsilon}_{\mathbf{2}}$ just after the transition has magnitude $\epsilon_{2} \simeq 0.01756$, and three possible orientations, i.e., monoclinic twins, defined by the phases, see Eq. (5),

$$
\phi_{2, n}=\frac{\pi}{6}+(n-1) \frac{2 \pi}{3}, \quad n=1,2,3 .
$$

In other words, $\boldsymbol{\epsilon}_{2}$ is directed along $\boldsymbol{b}_{m} \wedge \boldsymbol{c}_{H}$. The finite $\boldsymbol{\epsilon}_{2}$ in turns drives a finite $\boldsymbol{\epsilon}_{1}$, with magnitude 
$\epsilon_{1} \simeq 0.01337$ and angles,

$$
\phi_{1, n}=\left(2 \phi_{2, n}+\pi\right) \bmod (2 \pi) \text {, }
$$

implying that $\boldsymbol{\epsilon}_{1}$ is parallel to the monoclinic $\boldsymbol{b}_{m}$ axis, as well as finite $\epsilon \simeq 0.00937>0$ and $\epsilon_{3} \simeq-0.00487<0$.

Apart from assuming that the tilting and stretching of the $\mathrm{V}-\mathrm{V}$ dimers are distinct degrees of freedom, we shall here give up any attempt to describe microscopically the rhombohedral to monoclinic transition in $\mathrm{V}_{2} \mathrm{O}_{3}$, and instead resort to a more macroscopic approach based on a Landau-Ginzburg theory for the order parameter that ultimately characterises the structural transition, namely the shear strain $\epsilon_{2}$, and the one that instead drives the metal-insulator transition, i.e., the dimer length $d$.

\section{Landau-Ginzburg theory of the structural transition A Landau-Ginzburg free energy func-} tional for the space dependent order parameter $\epsilon_{2}(\mathbf{r})$ is rather cumbersome to derive, since all other components of the strain, beside the order parameter, are involved, and need to be integrated out to obtain a functional of $\epsilon_{2}(\mathbf{r})$ only. This is further complicated by the constraints due to the Saint-Venant compatibility equations, which avoid gaps and overlaps of different strained regions and play a crucial role in stabilising domains below the martensitic transformation ${ }^{59-61}$. Therefore, not to weigh down the text, we present the detailed derivation of the Landau-Ginzburg functional in the Supplementary Material ${ }^{43}$, and here just discuss the final result in the $c$-plane oriented film geometry of the experiments.

We find that the shear strain two-component order parameter $\epsilon_{2}(\mathbf{r})$ is controlled by the energy functional $^{43}$ 


$$
\begin{gathered}
E\left[\boldsymbol{\epsilon}_{2}\right]=\int d \mathbf{r}\left\{-\frac{K}{2} \boldsymbol{\epsilon}_{2}(\mathbf{r}) \cdot \nabla^{2} \boldsymbol{\epsilon}_{2}(\mathbf{r})+\tau \epsilon_{2}(\mathbf{r})^{2}-\gamma \epsilon_{2}(\mathbf{r})^{3} \sin 3 \phi_{2}(\mathbf{r})+\mu \epsilon_{2}(\mathbf{r})^{4}\right\} \\
+\kappa \iint d \mathbf{r} d \mathbf{r}^{\prime} \boldsymbol{\epsilon}_{2}(\mathbf{r}) \cdot \hat{U}\left(\mathbf{r}-\mathbf{r}^{\prime}\right) \boldsymbol{\epsilon}_{2}\left(\mathbf{r}^{\prime}\right),
\end{gathered}
$$

where $\mathbf{r}=r(\cos \phi, \sin \phi)$ is the two dimensional coordinate of the film, $\mu$ and $\kappa$ are positive parameters that depend on the elastic constants, while $\gamma$ is directly proportional to the elastic constant $c_{14}{ }^{43}$. The quadratic coupling constant $\tau$, which encodes the electronic effects, drives the transition and thus depends critically on temperature; it is positive in the corundum phase and negative in the monoclinic one. The last term in (9) derives from the Saint-Venant compatibility equations $^{43}$. Specifically,

$$
\hat{U}(\mathbf{r})=\left(\begin{array}{ll}
U_{11}(\mathbf{r}) & U_{12}(\mathbf{r}) \\
U_{21}(\mathbf{r}) & U_{22}(\mathbf{r})
\end{array}\right),
$$

where the long-range kernels have the explicit expressions:

$$
\begin{aligned}
& U_{11}(\mathbf{r})=-U_{22}(\mathbf{r})=-\frac{\cos 4 \phi}{\pi r^{2}}, \\
& U_{12}(\mathbf{r})=U_{21}(\mathbf{r})=\frac{\sin 4 \phi}{\pi r^{2}},
\end{aligned}
$$

and favour the existence of domains in the distorted structure ${ }^{59-61}$. The orientation of the interfaces between those domains is instead determined by the additional constraint we must impose ${ }^{43}$ to fulfil the Saint-Venant equations, namely the curl free condition

$$
\nabla \wedge \epsilon_{2}(\mathbf{r})=0
$$

If $\tau<0$, the energy (9) is minimum at the angles defined in Eq. (7) when $\epsilon_{2}>0$. The constraint (12) implies that a sharp interface between two domains, identified by two of the three 
possible directions of $\epsilon_{2}$, is oriented along the third one, namely along a mirror plane of the space group $R \overline{3} c$. This is exactly what is found experimentally, as we earlier discussed.

We also note that the minima of the energy functional (9) depend on the values and signs of $\tau$ and $\gamma \propto c_{14}$. Specifically, the corundum phase, $\epsilon_{2}=0$, is a local minimum for $\tau>\tau_{r} \gtrsim 0, \tau_{r}$ being the rhombohedral spinodal point. Similarly, a monoclinic phase is a local minimum for $\tau<\tau_{m}$, where the monoclinic spinodal point $\tau_{m} \geq \tau_{r}$. Phase coexistence thus occurs when $\tau \in\left[\tau_{r}, \tau_{m}\right]$, and also the structural transition must take place at $\tau_{c}$ within that same interval. At ambient temperature and pressure, $\tau \sim c_{44} \simeq 53 \mathrm{eV} / \mathrm{cm}^{3}$, which must be greater than $\tau_{c}$ since the stable phase is corundum, and $c_{14} \simeq-12.5 \mathrm{eV} / \mathrm{cm}^{3}{ }^{62-64}$, so that $\gamma$ is negative, too. Well below the structural transition at ambient pressure, $\tau$ must be smaller than $\tau_{r}$, and $\gamma$ has to become positive in order for $\boldsymbol{\epsilon}_{2}$ to have the observed values in the monoclinic phase. Therefore, upon lowering the temperature $T, \tau$ must decrease, while $c_{14} \propto \gamma$ must increase and cross zero. There are actually evidences, specifically in chromium doped compounds ${ }^{63}$, that $c_{14}$ does change sign approaching the transition from the corundum phase. We are not aware of any experimental measurement of $c_{14}$ in pure $\mathrm{V}_{2} \mathrm{O}_{3}$ below room temperature. Therefore, here we can only conjecture what may happen. One possibility is that $c_{14}$ crosses zero right at the structural transition, $\tau=\tau_{c}$. In such circumstance, the transition may become continuous ${ }^{65}$, which is likely the case of $\mathrm{Cr}$ doping with $x \gtrsim 0.03{ }^{66}$. The alternative compatible with the ambient pressure phase diagram in Fig. 1 for pure or weakly Ti/Cr doped $\mathrm{V}_{2} \mathrm{O}_{3}$ is that $c_{14}$ becomes positive at temperatures higher than $T_{c}$, thus the observed first order transition from the corundum phase, $\epsilon_{2}=0$, to the monoclinic one, $\epsilon_{2}>0$. There is still a third possibility that $\tau$ crosses $\tau_{c}$ at high temperature, when $c_{14} \propto \gamma$ is still negative. In such circumstance, the 
monoclinic phase that establishes for $\tau<\tau_{c}$ corresponds to a shear strain different from that observed in $\mathrm{V}_{2} \mathrm{O}_{3}$ at ambient pressure, specifically to a dimer tilting in the clockwise direction or, equivalently, to opposite $\epsilon_{2}$ vectors. This is presumably what happens in the monoclinic metal phase observed above $32.5 \mathrm{GPa}$ at $300 \mathrm{~K}^{38}$.

Here, we shall not consider such extreme conditions, and therefore take for granted that, at ambient pressure and for high temperatures such that $\gamma$ is negative, $\tau$ remains greater than $\tau_{c}$, so that the stable phase is always rhombohedral. Moreover, since we are just interested in pure $\mathrm{V}_{2} \mathrm{O}_{3}$, we shall assume that $\gamma$ becomes positive well above the structural transition, around which we shall therefore consider $\gamma>0$ constant, and $\tau \propto\left(T-T_{0}\right)$, with $T_{0}>0$ a parameter, playing the role of a reduced temperature.

Contribution from the dimer stretching The energy functional (9) controls only the shear strain $\epsilon_{2}$, namely the dimer tilting. We still need to include the dimer elongation, which is responsible of the metal-insulator transition ${ }^{44}$. Although the dimer length does not affect substantially the strain, the dimer tilting does favour its elongation, as discussed, e.g., by Tanaka in ${ }^{47}$. Following that approach, we assume that for a given tilting angle $\theta$, equivalently shear strain $\epsilon_{2}$, the dimer length $d$ has two equilibrium positions ${ }^{47}$. However, unlike Tanaka, we associate the two minima at shorter and longer $d$ in the metal and insulator phases, respectively. Therefore, replacing $d$ with the dimensionless space-dependent field $\eta(\mathbf{r})$, we assume the latter to be described by the energy 
functional

$$
\begin{aligned}
\delta E\left[\epsilon_{2}, \eta\right]=a \int d \mathbf{r}[ & \left(\eta(\mathbf{r})^{2}-\frac{1}{4}\right)^{2} \\
& \left.-g\left(\epsilon_{2}(\mathbf{r})^{2}-\epsilon_{I M T}^{2}\right) \eta(\mathbf{r})\right],
\end{aligned}
$$

with $a>0, g>0$, and $\epsilon_{I M T}^{2}$ either positive or negative. A minimum at $\eta<0$ corresponds to a metal, while one at $\eta>0$ to an insulator, and both may coexist. Note that $\epsilon_{2}^{2}>\epsilon_{I M T}^{2}$ implies that the global minimum is insulating, whereas $\epsilon_{2}^{2}<\epsilon_{I M T}^{2}$ that is metallic. In other words, $\epsilon_{I M T}^{2}$ represents the threshold strain above which an insulating phase becomes stable.

Total energy functional Adding the shear strain energy $E\left[\epsilon_{2}\right]$ in Eq. (9) to the dimer stretching contribution $\delta E\left[\epsilon_{2}, \eta\right]$ in Eq. (13), we obtain the total energy functional

$$
E\left[\boldsymbol{\epsilon}_{2}, \eta\right]=E\left[\boldsymbol{\epsilon}_{2}\right]+\delta E\left[\epsilon_{2}, \eta\right]
$$

which can actually describe rhombohedral and monoclinic phases, either metallic or insulating.

For instance, assuming $\epsilon_{I M T}^{2}<0$ in Eq. (13), the energy functional (14) predicts a transition from a rhombohedral insulator to a monoclinic one upon lowering $T$, i.e., the reduced temperature $\tau$, as indeed observed above $1 \%$ of $\mathrm{Cr}$ doping, see Fig. 1.

Pure $\mathrm{V}_{2} \mathrm{O}_{3}$ corresponds instead to assuming $\epsilon_{I M T}^{2}>0$. In this case, the stable rhombohedral phase, with $\epsilon_{2}=0$, is metallic. Upon crossing the first order structural transition at $T=T_{c}, \epsilon_{2}$ jumps directly to a finite value, $\epsilon_{2}\left(T_{c}\right)$, which grows upon further lowering $T$. If we assume, in agreement with the most recent experimental claims ${ }^{58}$, that at equilibrium there is no monoclinic metal phase in between the rhombohedral metal and monoclinic insulator, we must conclude that 
just after the transition to the monoclinic phase $\epsilon_{2}\left(T_{c}\right)^{2}-\epsilon_{I M T}^{2}>0$, so that the global monoclinic minimum is always insulating. Consequently, we fixed the parameters of the energy functional so that, assuming homogeneous phases, i.e., neglecting the Ginzburg term and the long range potential, $K=\kappa=0$ in Eq. (9), the phase diagram, see Fig. 7, shows a direct first order transition from a corundum metal to a monoclinic insulator, with a coexistence region of width $\Delta T=\Delta \tau \simeq$ $40 \mathrm{~K}$ consistent with experiments. We emphasise that the absence of a stable monoclinic metal does not exclude its presence as a metastable phase that is allowed by the energy functional (14), and which we indeed find, see Fig. 7.

Domains at equilibrium We mentioned that the long range elastic potential $\hat{U}(\mathbf{r})$ in $(9)$ favours 59 the existence of domains in the lower symmetry monoclinic phase, which seem to persist also within the insulator-metal coexistence region across the first order transition ${ }^{35,37,57,58}$. Since the configurational entropy in presence of different domains plays an important role at finite temperature, we cannot simply search for the minima of the classical energy functional Eq. (14) varying the reduced temperature $\tau$, as in Fig. 7, but we need to calculate actual thermodynamic averages. For that, we take inspiration from the mean field theory developed in Refs. 61, 67, which was orginally developed for ferroelastic transitions, and we extend it to treat the lattice and electronic insulator-to-metal nanotextured dynamics in a Mott material. We discuss thoroughly such meanfield scheme in the Supplementary Material ${ }^{43}$, while here we just present the results.

In Fig. 8 we show the calculated real space distribution of the shear strain $\epsilon_{2}(\mathbf{r})$ at low temperature, i.e., deep inside the monoclinic insulator. As expected, the distribution is not homo- 
geneous, but shows coexistence of equivalent monoclinic twins, each characterised by a colour that corresponds to one of the three equivalent shear strain vectors $\boldsymbol{\epsilon}_{2, i}, i=1,2,3$, see Eq. (7), which are shown on the left in Fig. 8. We note that the interface between two domains, i.e., two strain vectors $\boldsymbol{\epsilon}_{2, i}$ and $\boldsymbol{\epsilon}_{2, j}, i \neq j$, is directed along the third vector, $\boldsymbol{\epsilon}_{2, k}, k \neq i, j$, in accordance with the curl-free condition (12), and with the experimental data presented in Sec. 2. In addition, the strain along the interfaces is strongly suppressed as compared to the interior of each domain, as indicated by the lighter regions in Fig. 8. The inherent suppression of the strain amplitude at the domain boundaries will turn out to play a fundamental role in seeding and stabilizing the photo-induced non-thermal metallic phase.

In the top panel of Fig. 9 we show the calculated real space distribution of $\epsilon_{2}(\mathbf{r})$ within the monoclinic-rhombohedral coexistence region across the temperature driven first-order transition, where the green colour indicates the rhombohedral domains. The bottom panel of Fig. 9 instead shows the real space distribution of $\epsilon_{2}(\mathbf{r})^{2}=\boldsymbol{\epsilon}_{2}(\mathbf{r}) \cdot \boldsymbol{\epsilon}_{2}(\mathbf{r})$, which is finite in any monoclinic domain, and zero in rhombohedral ones. We mentioned that the insulator is locally stable if the shear-strain amplitude square $\epsilon_{2}(\mathbf{r})^{2}>\epsilon_{I M T}^{2}$, otherwise the locally stable phase is metallic. For that reason, we use in the bottom panel of Fig. 9 a blue colorscale for all regions where $\epsilon_{2}(\mathbf{r})^{2}>\epsilon_{I M T}^{2}$, and a red colorscale for $\epsilon_{2}(\mathbf{r})^{2}<\epsilon_{I M T}^{2}$, the two colours thus distinguishing between insulating and metallic domains. We note that, as $T$ raises, metallic domains start to nucleate first with a residual monoclinic strain, light red, that soon disappears, dark red. This gives evidence that the metastable monoclinic metal does appear across the monoclinic-rhombohedral phase transition, even though it gives in to the rhombohedral metal before a percolating metal cluster first sets in, in accordance 
with experiments ${ }^{58}$.

We also observe that rhombohedral domains, dark red, have triangular shapes, as dictated by the curl-free condition (12) at the interfaces between monoclinic and rhombohedral domains. This pattern does not resemble the observed experimental one ${ }^{35}$. This difference is due to the $c$-plane orientation that we use, in contrast to the A-plane one in the experiment ${ }^{35}$.

\section{Light-induced non-equilibrium transition: evidence of a transient monoclinic metal phase}

Space-dependent non-equilibrium model We note that the above theoretical modelling may also account for the experimental evidences ${ }^{9,20,21,37}$ of a photo-induced metal phase in $\mathrm{V}_{2} \mathrm{O}_{3}$. As discussed in Refs. 20,21,37, the main effect of the $1.5 \mathrm{eV}$ laser pulse is to transfer electrons from the $e_{g}^{\pi}$ to the $a_{1 g}$ derived bands, see Fig. 3. The increase in $a_{1 g}$ population at the expense of the $e_{g}^{\pi}$ one leads to a transient reduction of the actual trigonal field splitting between $e_{g}^{\pi}$ and $a_{1 g}$ orbitals, which depends on their occupations via Coulomb interaction, thus driving the insulator towards the metal. Such effect of the laser pump can be easily included in the double-well potential (13) that describes the dimer stretching, i.e., the trigonal splitting, by adding a laser fluence, $f$, dependent term linear in $\eta$, namely

$$
\delta E\left[\epsilon_{2}, \eta\right] \rightarrow \delta E\left[\epsilon_{2}, \eta\right]+\int d \mathbf{r} \mu(f) \eta(\mathbf{r})
$$

with $\mu(f)>0$, being zero at $f=0$ and growing with it, thus favouring the metal state with $\eta<0$. This term is actually equivalent to a fluence dependent threshold strain

$$
\epsilon_{I M T}^{2} \rightarrow \epsilon_{I M T}^{2}(f) \equiv \epsilon_{I M T}^{2}+\mu(f) / g
$$


that increases with $f$. Looking at the bottom panel of Fig. 9, such upward shift of $\epsilon_{I M T}(f)$ implies not only that formerly insulating regions with $\epsilon_{I M T}<\epsilon_{2}(\mathbf{r})<\epsilon_{I M T}(f)$ may turn metallic, but also the possibility that a laser pulse with a fluence exceeding a threshold value stabilises the formerly metastable monoclinic metal. Indeed, we earlier mentioned that we fix $\epsilon_{I M T}$ smaller than the shear strain amplitude $\epsilon_{2}\left(T_{c}\right)$ just after the rhombohedral to monoclinic first order transition, which ensures the absence of a stable monoclinic metal at equilibrium. After laser irradiation, $\epsilon_{I M T}(f)$ may well surpass the shear strain amplitude, in turn reduced by heating effects, when $f$ exceeds a threshold fluence, thus stabilising the monoclinic metal, metastable at equilibrium.

In order to simulate the spatial dynamics of the laser-induced metalization, we start from the calculated shear strain map, reported in Fig. 8. As the pump excitation fluence rises, the concurrent increase of $\epsilon_{I M T}(f)$ leads to the possible nucleation of non-thermal metal regions with finite monoclinic shear strain, whenever the condition $\epsilon_{2}(\mathbf{r}) \leq \epsilon_{I M T}(f)$ is met. In Fig. 10 we report the spatial configuration of such domains (purple areas), which are metallic and yet characterized by the same in-plane monoclinic nanotexture of the insulating phase (see Supplementary Material ${ }^{43}$ for the parameters). We note that the non-thermal metal starts nucleating at the boundaries between different monoclinic twins, where, as previously discussed, the strain is constrained to smaller values than in the interior of each domain. As $\epsilon_{I M T}(f)$ increases, the filling fraction of the non-thermal metal phase grows progressively up to the point of occupying the entire region.

Time-resolved PEEM experiments To demonstrate that such intriguing scenario indeed realises in photoexcited $\mathrm{V}_{2} \mathrm{O}_{3}$, we developed a novel time-resolved X-ray PEEM experiment (see Fig. 11) 
with $30 \mathrm{~nm}$ and 80 ps spatial and temporal resolution (see Supplementary Material ${ }^{43}$ for the experimental details). With this imaging method we studied the temporal response of the monoclinic domains triggered by a properly synchronized pulsed laser excitation (1.5 eV photon energy; $\sim 50$ fs pulse duration) capable of impulsively changing the $e_{g}^{\pi}$ and $a_{1 g}$ band population and possibly inducing the non-thermal metallic monoclinic state.

The time-resolved experiments were performed on a $50 \mathrm{~nm} \mathrm{~V}_{2} \mathrm{O}_{3}$ crystalline film deposited by oxygen-assisted molecular beam epitaxy on a sapphire substrate, with $c$-axis perpendicular to the surface ${ }^{45}$. In order to characterize the IMT, we first measured the temperature-dependent optical properties at a selected probe photon energy $(2.4 \mathrm{eV})$ during the heating and cooling cycles. The curve reported in Fig. 12a shows the typical hysteresis of the insulator to metal transition with mid-point at $T_{c} \simeq 140 \mathrm{~K}$, slightly smaller than that observed in bulk crystals as a consequence of the film residual strain ${ }^{45}$. The reflectivity at $2.4 \mathrm{eV}$ drops by $14 \%$ when the temperature is increased from $100 \mathrm{~K}$ (insulating phase) to $180 \mathrm{~K}$ (metallic phase), while the film resistivity drops by approximately 3 orders of magnitude (see Supplementary Figure S2 ${ }^{43}$ ). Figs. 12 b and 12 c show equilibrium XLD-PEEM images of the sample taken at $T=100 \mathrm{~K}$ and $180 \mathrm{~K}$, respectively. As discussed at length in Sec. 2 the XLD-PEEM images clearly evidence in the low temperature monoclinic phase the formation of stripe-like domains corresponding to different monoclinic twins 37. When the temperature is increased well above $T_{c}$, the monoclinic nanotexture is replaced by a homogeneous corundum phase with almost absent XLD contrast.

As extensively discussed in the literature $20,21,27,28,37,68,69$, the electronic IMT can be also 
photo-induced by using ultrashort infrared pulses as the external control parameter. When the excitation is intense enough, the insulating phase collapses on a timescale of $\sim 30-50$ ps transforming into a new phase with the same optical properties as the metallic one. We monitored such transformation in our sample by recording the relative reflectivity variation after 100 ps between the optical pump and probe, i.e. when the time-resolved signal already reached a plateau (see Figure $\mathrm{S} 3)$. In Fig. 12d we show the relative reflectivity variation at $2.4 \mathrm{eV}$ probe photon energy as a function of the impinging pump fluence. Above $\approx 8 \mathrm{~mJ} / \mathrm{cm}^{2}$, the measured reflectivity drop perfectly matches the equilibrium reflectivity difference between the insulating and metallic phases, thus demonstrating that the whole of the pumped volume is turned into the electronic metallic phase.

In order to investigate the dynamics of the monoclinic domains during the photoinduced insulator-to-metal transition, we performed a XLD-PEEM experiment exploiting the inherent pulse structure of the synchrotron X-ray radiation and the synchronization with a femtosecond laser source, which allows turning the XLD-PEEM experiment into a time-resolved microscopy tool with 80 ps time-resolution (see Supplementary Material ${ }^{43}$ ). The experiment was carried out on the same sample, and in the same experimental conditions as the optical pump-probe results reported in Fig. 12d, in order to avoid possible artefacts related to different average heating in the two experiments ${ }^{30}$. The pump-probe spatial and temporal overlap was carefully checked by exploiting the non-linear photoemision from surface impurities, as explained in detail in the Supplementary Material $^{43}$ (see Sec. S1). Fig. 13a displays a typical image of the monoclinic domains 150 ps before and 30 ps after the excitation with laser pulses at $22 \pm 4 \mathrm{~mJ} / \mathrm{cm}^{2}$ fluence. Although the excitation exceeds by far the threshold necessary to photoinduce the complete transformation into 
the electronic metallic phase, our results show that, despite a moderate smearing of the contrast due to a small residual average heating, the monoclinic nanotexture is fully retained. These results demonstrate that, upon photoexcitation, the system undergoes an electronic transformation from the insulating to a metal-like state, while maintaining the in-plane shear strain of the monoclinic phase. In Fig. 13c we report the long-time dynamics of the contrast between neighbouring domains as a function of the time delay between the infrared pump and the X-ray probe. The photoinduced signal recovers within 3 ns, which corresponds to the cooling time of the sample ${ }^{9}$. No signature of long-time melting of the monoclinic nanotexture is observed.

A possible route to control the non-thermal metallic phase is given by interface strain engineering ${ }^{70}$, which allows to control the residual interface strain in the $\mathrm{V}_{2} \mathrm{O}_{3}$ film. Quite naturally, the presence of residual tensile strain in the film may enhance $\epsilon_{I M T}$ and favour the emergence of monoclinic metallic regions. In Fig. 14a we compare the fluence dependent filling fraction measured on the $\mathrm{V}_{2} \mathrm{O}_{3}$ film used for tr-PEEM measurements to that obtained on a similar $\mathrm{V}_{2} \mathrm{O}_{3}$ film in which the residual strain is diminished by means of $\mathrm{C}_{2} \mathrm{O}_{3}$ buffer layer (see Section $\mathrm{S} 2$ of the Supplementary Material $\left.{ }^{43}\right)^{45}$. The fluence dependent data show a remarkably different metallization dynamics, compatible with a decrease of $\epsilon_{I M T}$ in the film with the $\mathrm{Cr}_{2} \mathrm{O}_{3}$ buffer layer. We underline that, in both cases, the morphology of monoclinic domains is very similar, as shown by PEEM images (see Fig. S4). The difference in the $\epsilon_{I M T}$ values does not impact on the monoclinic nanotexture, which is governed by the functional (9), but rather controls the fragility toward the emergence of the photo-induced non-thermal metallic phase. In Fig. 14b we present more data points showing the correlation between residual tensile strain and the value of $\epsilon_{I M T}$. 


\section{Conclusions}

In this work, we have developed a coarse-grained model, based on the minimization of a LandauGinzburg energy functional, to account for the space-dependent lattice and electronic dynamics across the insulator-to-metal transition in the archetypal Mott insulator $\mathrm{V}_{2} \mathrm{O}_{3}$. The spontaneous long-range nanotexture, originated by the minimization of the lattice energy, emerges as a key element to describe both the temperature-driven and the photoinduced transition. The reduced-strain regions at the domain boundaries and corners provide the necessary template for the nucleation of metallic domains. In out-of-equilibrium conditions, the domain boundaries stabilize and protect the photoinduced non-thermal monoclinic metallic state, which would be unstable at equilibrium an in homogeneous systems.

Although the reported theory and experiments refer to the Mott transition in $\mathrm{V}_{2} \mathrm{O}_{3}$, the present results unveil a profound and general link between the real-space topology, the transition dynamics and the emergence of non-thermal electronic states in quantum materials. The combination of multiscale modeling and microscopy experiments with time-resolution offers new platforms to understand and control the transition dynamics of solids which exhibit spontaneous self-organization at the nanoscale. Indeed the complexity of space-dependent sold-solid phase transformations involving different degrees of freedom (electrons, lattice, spins, etc.) opens new exciting possibilities for achieving the full control of the transition and for synthesizing novel emerging metastable states that do not exist at equilibrium and in homogeneous phases. The combination of real-space morphology control via interface engineering, electric fields or pressure, 
with the development of novel excitation schemes to coherently manipulate insulator to metal phase transitions ${ }^{71}$ are expected to open new routes for achieving the full and reversible control of the electronic properties of correlated oxides.

From the theoretical side, our results call for the development of realistic models that capture the long-range dynamics and complexity of electronic transitions, which are usually tackled starting from a microscopic approach. Experimentally, the present results justify the ongoing efforts to develop novel table-top and large-scale facility time-resolved microscopy techniques to investigate the intertwining between non-thermal properties and real-space morphology in quantum correlated materials. Addressing the role of real space inhomogeneities and intrinsic strain nanotexture will be crucial to finally clarify the long-standing issue about the possibility of fully decoupling and control the electronic and structural phase transitions in vanadates ${ }^{3,25,26,29,30,69,72-74}$ and other Mott materials.

\section{Acknowledgments}

C.G., A.R. and P.F. acknowledge financial support from MIUR through the PRIN 2015 (Prot. 2015C5SEJJ001) and PRIN 2017 (Prot. 20172H2SC4_005) programs. C.G. and G.F. acknowledge support from Università Cattolica del Sacro Cuore through D.1, D.2.2, and D.3.1 grants. We acknowledge Diamond Light Source for the provision of beamtime under proposals number SI18897 and MM21700. JPL acknowledges financial supported by the KU Leuven Research Funds, Project No. KAC24/18/056, No. C14/17/080 and iBOF/21/084 as well as the Research 
Funds of the INTERREG-E-TEST Project (EMR113) and INTERREG-VL-NL-ETPATHFINDER

Project (0559). M.M. acknowledges support from "Severo Ochoa" Programme for Centres of Excellence in R\&D (MINCINN, Grant SEV-2016-0686). M.F. has received funding from the European Research Council (ERC) under the European Union's Horizon 2020 research and innovation programme, Grant agreement No. 692670 "FIRSTORM".

1. Mott, N. F. The basis of the electron theory of metals, with special reference to the transition metals. Proceedings of the Physical Society. Section A 62, 416 (1949). URL http://stacks.iop. org/0370-1298/62/i=7/a=303.

2. Fish, J., Wagner, G. J. \& Keten, S. Mesoscopic and multiscale modelling in materials. Nature Materials 20, 774-786 (2021).

3. McLeod, A. S. et al. Nanotextured phase coexistence in the correlated insulator $\mathrm{V}_{2} \mathrm{O}_{3}$. Nature Physics 13, 80 (2016).

4. McLeod, A. S. et al. Nano-imaging of strain-tuned stripe textures in a Mott crystal. npj Quantum Materials 6, 46 (2021).

5. Ronchi, A. et al. Ultrafast orbital manipulation and Mott physics in multi-band correlated materials. Proc. SPIE 17 (2018).

6. Stoliar, P. et al. Universal Electric-Field-Driven Resistive Transition in Narrow-Gap Mott Insulators. Advanced Materials 25, 3222-3226 (2013). 
7. Stoliar, P. et al. A Leaky-Integrate-and-Fire Neuron Analog Realized with a Mott Insulator. Advanced Functional Materials 27, 1604740 (2017).

8. del Valle, J., Ramirez, J. G., Rozenberg, M. J. \& Schuller, I. K. Challenges in materials and devices for resistive-switching-based neuromorphic computing. Journal of Applied Physics 124, 211101 (2018).

9. Ronchi, A. et al. Light-Assisted Resistance Collapse in a $\mathrm{V}_{2} \mathrm{O}_{3}$-Based Mott-Insulator Device. Phys. Rev. Applied 15, 044023 (2021).

10. Adda, C. et al. Mott insulators: A large class of materials for Leaky Integrate and Fire (LIF) artificial neuron. Journal of Applied Physics 124, 152124 (2018).

11. Adda, C. et al. First demonstration of "Leaky Integrate and Fire" artificial neuron behavior on (V0.95Cr0.05)2O3 thin film. MRS Communications 8, 835-841 (2018).

12. Pérez-Tom1'as, A. Functional Oxides for Photoneuromorphic Engineering: Toward a Solar Brain. Advanced Materials Interfaces 6, 1900471 (2019).

13. Kim, H., Jo, Y., Kim, S., Kim, D. \& Hur, J. 2g hts racetrack coil protection using smart switching feature of v2o3. IEEE Transactions on Applied Superconductivity 29, 1-5 (2019).

14. Salev, P., del Valle, J., Kalcheim, Y. \& Schuller, I. K. Giant nonvolatile resistive switching in a mott oxide and ferroelectric hybrid. Proceedings of the National Academy of Sciences 116, 8798-8802 (2019). 
15. del Valle, J. et al. Subthreshold firing in mott nanodevices. Nature 569, 388-392 (2019). URL https://doi.org/10.1038/s41586-019-1159-6.

16. Stoliar, P. et al. Universal Electric-Field-Driven Resistive Transition in Narrow-Gap Mott Insulators. Advanced Materials 25, 3222-3226 (2013).

17. Rupp, J. A. J. et al. Different threshold and bipolar resistive switching mechanisms in reactively sputtered amorphous undoped and Cr-doped vanadium oxide thin films. Journal of Applied Physics 123, 044502 (2018).

18. Rúa, A. et al. Toward reproducible metal-insulator transition characteristics in $\mathrm{V}_{2} \mathrm{O}_{3}$ thin films sputter-deposited on glass. Journal of Applied Physics 124, 205301 (2018).

19. Wouters, D. J., Menzel, S., Rupp, J. A. J., Hennen, T. \& Waser, R. On the universality of the i-v switching characteristics in non-volatile and volatile resistive switching oxides. Faraday Discuss. 213, 183-196 (2019). URL http://dx.doi.org/10.1039/C8FD00116B.

20. Lantz, G. et al. Ultrafast evolution and transient phases of a prototype out-of-equilibrium Mott-Hubbard material. Nature Communications 8, 13917 (2017). URL https://doi.org/10. 1038/ncomms13917.

21. Boschetto, D. et al. Reply to: Ultrafast evolution and transient phases of a prototype out-ofequilibrium Mott-Hubbard material. Nature Communications 10, 4035 (2019). URL https: //doi.org/10.1038/s41467-019-11744-2. 
22. Giorgianni, F., Sakai, J. \& Lupi, S. Overcoming the thermal regime for the electric-field driven Mott transition in vanadium sesquioxide. Nature Communications 10, 1159 (2019). URL https://doi.org/10.1038/s41467-019-09137-6.

23. Kalcheim, Y. et al. Non-thermal resistive switching in Mott insulator nanowires. Nature Communications 11, 2985 (2020).

24. Giorgianni, F., Sakai, J. \& Lupi, S. Overcoming the thermal regime for the electric-field driven Mott transition in vanadium sesquioxide. Nature Communications 10, 1159 (2019).

25. Morrison, V. R. et al. A photoinduced metal-like phase of monoclinic $\mathrm{VO}_{2}$ revealed by ultrafast electron diffraction. Science 346, 445-448 (2014).

26. Otto, M. R. et al. How optical excitation controls the structure and properties of vanadium dioxide. Proceedings of the National Academy of Sciences 116, 450-455 (2019).

27. Abreu, E. et al. Dynamic conductivity scaling in photoexcited $\mathrm{V}_{2} \mathrm{O}_{3}$ thin films. Phys. Rev. B 92, $085130(2015)$.

28. Abreu, E. et al. Ultrafast electron-lattice coupling dynamics in $\mathrm{VO}_{2}$ and $\mathrm{V}_{2} \mathrm{O}_{3}$ thin films. Phys. Rev. B 96, 094309 (2017).

29. Lantz, G. et al. Ultrafast evolution and transient phases of a prototype out-of-equilibrium Mott-Hubbard material. Nature Communications 8, 13917 (2017).

30. Vidas, L. et al. Does $\mathrm{VO}_{2}$ Host a Transient Monoclinic Metallic Phase? Phys. Rev. X 10, 031047 (2020). 
31. McWhan, D. B., Rice, T. M. \& Remeika, J. B. Mott transition in Cr-doped $\mathrm{V}_{2} \mathrm{O}_{3}$. Phys. Rev. Lett. 23, 1384 (1969).

32. McWhan, D. B. \& Remeika, J. P. Metal-insulator transition in $\left(\mathrm{V}_{1-x} \mathrm{Cr}_{x}\right)_{2} \mathrm{O}_{3}$. Phys. Rev. B 2, 3734 (1970).

33. McWhan, D. B. et al. Electronic specific heat of metallic Ti-doped $\mathrm{V}_{2} \mathrm{O}_{3}$. Phys. Rev. Lett. 27, $941(1971)$.

34. McWhan, D. B., Menth, A., Remeika, J. P., Rice, T. M. \& Brinkman, W. F. Metal-insulator transitions in pure and doped $\mathrm{V}_{2} \mathrm{O}_{3}$. Phys. Rev. B 7, 1920 (1973).

35. McLeod, A. S. et al. Nanotextured phase coexistence in the correlated insulator $\mathrm{V}_{2} \mathrm{O}_{3}$. Nature Physics 13, 80-86 (2017). URL https://doi.org/10.1038/nphys3882.

36. Shao, Z. Lattice Distortion of $\mathrm{V}_{2} \mathrm{O}_{3}$ thin films during metal-to-insulator transition. Master's thesis, Cornell University (2019). URL https://hdl.handle.net/1813/67719.

37. Ronchi, A. et al. Early-stage dynamics of metallic droplets embedded in the nanotextured Mott insulating phase of $\mathrm{V}_{2} \mathrm{O}_{3}$. Phys. Rev. B 100, 075111 (2019). URL https://link.aps.org/ doi/10.1103/PhysRevB.100.075111.

38. Ding, Y. et al. Novel High-Pressure Monoclinic Metallic Phase of $\mathrm{V}_{2} \mathrm{O}_{3}$. Phys. Rev. Lett. 112, 056401 (2014). URL https://link.aps.org/doi/10.1103/PhysRevLett.112.056401.

39. Bai, L. et al. Pressure-induced cation-cation bonding in $\mathrm{V}_{2} \mathrm{O}_{3}$. Phys. Rev. B 92, 134106 (2015). URL https://link.aps.org/doi/10.1103/PhysRevB.92.134106. 
40. Chudnovskii, F. et al. Acoustic Emission during Metal-Insulator Phase Transition in $\mathrm{V}_{2} \mathrm{O}_{3}$. Journal of Solid State Chemistry 133, 430 - 433 (1997). URL http://www.sciencedirect.com/ science/article/pii/S0022459697975034.

41. Dernier, P. D. \& Marezio, M. Crystal structure of the low-temperature antiferromagnetic phase of $\mathrm{V}_{2} \mathrm{O}_{3}$. Phys. Rev. B 2, 3771 (1970).

42. Fabrykiewicz, P., Przeniosło, R., Sosnowska, I. \& Fauth, F. Positive and negative monoclinic deformation of corundum-type trigonal crystal structures of $M_{2} \mathrm{O}_{3}$ metal oxides. Acta Crystallographica Section B 74, 660-672 (2018). URL https://doi.org/10.1107/S2052520618014968.

43. See the Supplementary Material at....

44. Poteryaev, A. I. et al. Enhanced crystal field splitting and orbital selective coherence by strong correlations in $\mathrm{V}_{2} \mathrm{O}_{3}$. Phys. Rev. B 76, 085127 (2007).

45. Dillemans, L. et al. Evidence of the metal-insulator transition in ultrathin unstrained $\mathrm{V}_{2} \mathrm{O}_{3}$ thin films. Applied Physics Letters 104, 071902 (2014).

46. Scholl, A. et al. Observation of Antiferromagnetic Domains in Epitaxial Thin Films. Science 287, 1014-1016 (2000).

47. Tanaka, A. Electronic Structure and Phase Transition in $\mathrm{V}_{2} \mathrm{O}_{3}$ : Importance of $3 \mathrm{~d}$ Spin-Orbit Interaction and Lattice Distortion. Journal of the Physical Society of Japan 71, 1091 (2002). 
48. Grieger, D. \& Fabrizio, M. Low-temperature magnetic ordering and structural distortions in vanadium sesquioxide $\mathrm{V}_{2} \mathrm{O}_{3}$. Phys. Rev. B 92, 075121 (2015). URL https://link.aps.org/doi/ 10.1103/PhysRevB.92.075121.

49. Fouet, J., Sindzingre, P. \& Lhuillier, C. An investigation of the quantum $J_{1}-J_{2^{-}} J_{3}$ model on the honeycomb lattice. The European Physical Journal B - Condensed Matter and Complex Systems 20, 241 (2001).

50. Albuquerque, A. et al. Phase diagram of a frustrated quantum antiferromagnet on the honeycomb lattice: Magnetic order versus valence-bond crystal formation. Phys. Rev. B 84, 024406 (2011).

51. Mezzacapo, F. \& Boninsegni, M. Ground-state phase diagram of the quantum $J_{1}-J_{2}$ model on the honeycomb lattice. Phys. Rev. B 85, 060402 (2012).

52. Leiner, J. C. et al. Frustrated Magnetism in Mott Insulating $\left(\mathrm{V}_{1-x} \mathrm{Cr}_{x}\right)_{2} \mathrm{O}_{3}$. Phys. Rev. $X$ 9, 011035 (2019). URL https://link.aps.org/doi/10.1103/PhysRevX.9.011035.

53. Pfalzer, P., Obermeier, G., Klemm, M., Horn, S. \& denBoer, M. L. Structural precursor to the metal-insulator transition in $\mathrm{V}_{2} \mathrm{O}_{3}$. Phys. Rev. B 73, 144106 (2006). URL https://link.aps.org/ doi/10.1103/PhysRevB.73.144106.

54. Kündel, J. et al. Direct observation of the lattice precursor of the metal-to-insulator transition in $\mathrm{V}_{2} \mathrm{O}_{3}$ thin films by surface acoustic waves. Applied Physics Letters 102, 101904 (2013).

55. Majid, S. S. et al. Stabilization of metallic phase in $\mathrm{V}_{2} \mathrm{O}_{3}$ thin film. Applied Physics Letters 110, 173101 (2017). 
56. Singer, A. et al. Nonequilibrium Phase Precursors during a Photoexcited Insulator-to-Metal Transition in $\mathrm{V}_{2} \mathrm{O}_{3}$. Phys. Rev. Lett. 120, 207601 (2018). URL https://link.aps.org/doi/10. 1103/PhysRevLett.120.207601.

57. Frandsen, B. A. et al. Intertwined magnetic, structural, and electronic transitions in $\mathrm{V}_{2} \mathrm{O}_{3}$. Phys. Rev. B 100, 235136 (2019). URL https://link.aps.org/doi/10.1103/PhysRevB.100. 235136.

58. Kalcheim, Y. et al. Robust Coupling between Structural and Electronic Transitions in a Mott Material. Phys. Rev. Lett. 122, 057601 (2019).

59. Rasmussen, K. O. et al. Three-Dimensional Elastic Compatibility and Varieties of Twins in Martensites. Phys. Rev. Lett. 87, 055704 (2001). URL https://link.aps.org/doi/10.1103/ PhysRevLett.87.055704.

60. Shenoy, S. R., Lookman, T., Saxena, A. \& Bishop, A. R. Martensitic textures: Multiscale consequences of elastic compatibility. Phys. Rev. B 60, R12537-R12541 (1999). URL https: //link.aps.org/doi/10.1103/PhysRevB.60.R12537.

61. Vasseur, R., Lookman, T. \& Shenoy, S. R. Microstructure from ferroelastic transitions using strain pseudospin clock models in two and three dimensions: A local mean-field analysis. Phys. Rev. B 82, 094118 (2010). URL https://link.aps.org/doi/10.1103/PhysRevB.82.094118.

62. Nichols, D. N., Sladek, R. J. \& Harrison, H. R. Elastic constants of $\mathrm{V}_{2} \mathrm{O}_{3}$ between 300 and 640 K: Anomalies near the high-temperature electrical transition. Phys. Rev. B 24, 3025-3030 (1981). URL https://link.aps.org/doi/10.1103/PhysRevB.24.3025. 
63. Yang, H., Sladek, R. \& Harrison, H. Shear elastic constant softening in $\left.\mathrm{V}_{1-x} \mathrm{Cr}_{x}\right)_{2} \mathrm{O}_{3}$ : Secondorder nature of the low temperature phase transition. Solid State Communications 47, 955 957 (1983). URL http://www.sciencedirect.com/science/article/pii/0038109883909778.

64. Yang, H. \& Sladek, R. J. Elastic anomalies in $\left(\mathrm{V}_{1-\mathrm{x}} \mathrm{Cr}_{\mathrm{x}}\right)_{2} \mathrm{O}_{3}$ : Electron-transfer effects. Phys. Rev. B 34, 2627-2631 (1986). URL https://link.aps.org/doi/10.1103/PhysRevB.34.2627.

65. Boccara, N. Second-order phase transitions characterized by a deformation of the unit cell. Annals of Physics 47, 40 - 64 (1968). URL http://www.sciencedirect.com/science/article/pii/ $000349166890225 X$

66. Yethiraj, M., Werner, S. A., Yelon, W. B. \& Honig, J. M. Phonon anomalies and the magnetic transition in pure and Cr-doped $\mathrm{V}_{2} \mathrm{O}_{3}$. Phys. Rev. B 36, 8675-8686 (1987). URL https: //link.aps.org/doi/10.1103/PhysRevB.36.8675.

67. Shenoy, S. R. \& Lookman, T. Strain pseudospins with power-law interactions: Glassy textures of a cooled coupled-map lattice. Phys. Rev. B 78, 144103 (2008). URL https://link.aps.org/ doi/10.1103/PhysRevB.78.144103.

68. Liu, M. K. et al. Photoinduced Phase Transitions by Time-Resolved Far-Infrared Spectroscopy in $\mathrm{V}_{2} \mathrm{O}_{3}$. Phys. Rev. Lett. 107, 066403 (2011).

69. Singer, A. et al. Nonequilibrium Phase Precursors during a Photoexcited Insulator-to-Metal Transition in $\mathrm{V}_{2} \mathrm{O}_{3}$. Phys. Rev. Lett. 120, 207601 (2018). 
70. Homm, P., Menghini, M., Seo, J. W., Peters, S. \& Locquet, J. P. Room temperature Mott metal-insulator transition in $\mathrm{V}_{2} \mathrm{O}_{3}$ compounds induced via strain-engineering. APL Materials 9, 021116 (2021).

71. Horstmann, J. G. et al. Coherent control of a surface structural phase transition. Nature 583, 232-236 (2020).

72. Nájera, O., Civelli, M., Dobrosavljević, V. \& Rozenberg, M. J. Resolving the $\mathrm{VO}_{2}$ controversy: Mott mechanism dominates the insulator-to-metal transition. Phys. Rev. B 95, 035113 (2017).

73. Kalcheim, Y. et al. Robust Coupling between Structural and Electronic Transitions in a Mott Material. Phys. Rev. Lett. 122, 057601 (2019).

74. Frandsen, B. A. et al. Intertwined magnetic, structural, and electronic transitions in $\mathrm{V}_{2} \mathrm{O}_{3}$. Phys. Rev. B 100, 235136 (2019).

75. McWhan, D. B. et al. Electronic specific heat of metallic ti-doped $\mathrm{v}_{2} \mathrm{O}_{3}$. Phys. Rev. Lett. 27, 941-943 (1971). URL https://link.aps.org/doi/10.1103/PhysRevLett.27.941.

76. Park, J.-H. et al. Spin and orbital occupation and phase transitions in $\mathrm{V}_{2} \mathrm{O}_{3}$. Phys. Rev. B 61, 11506-11509 (2000). 


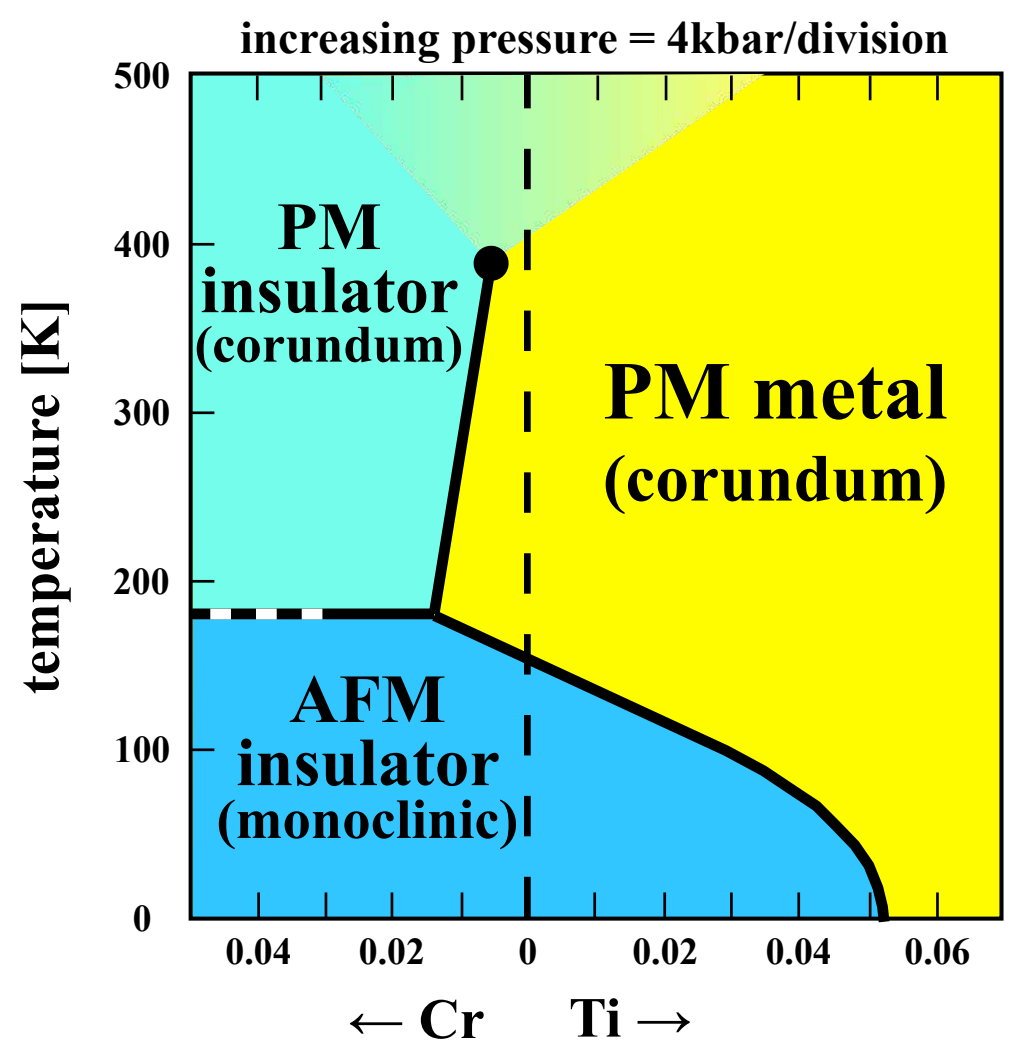

Figure 1: Phase diagram of $\left(\mathrm{V}_{1-x} \mathrm{M}_{x}\right)_{2} \mathrm{O}_{3}$, with $\mathrm{M}=\mathrm{Cr}$, Ti, as function of the doping concentration $x$ and pressure, from Ref. ${ }^{75}$. AFM and PM stand for antiferromagnetic and paramagnetic, respectively. All transition lines (solid black lines) are first order, with the one separating PM metal from PM insulator that terminates into a second order critical point (black dot). For Cr doping $x \gtrsim 0.03$ there are evidences ${ }^{66}$ that the transition becomes continuous, or very weakly first order, thus the dashed line. 


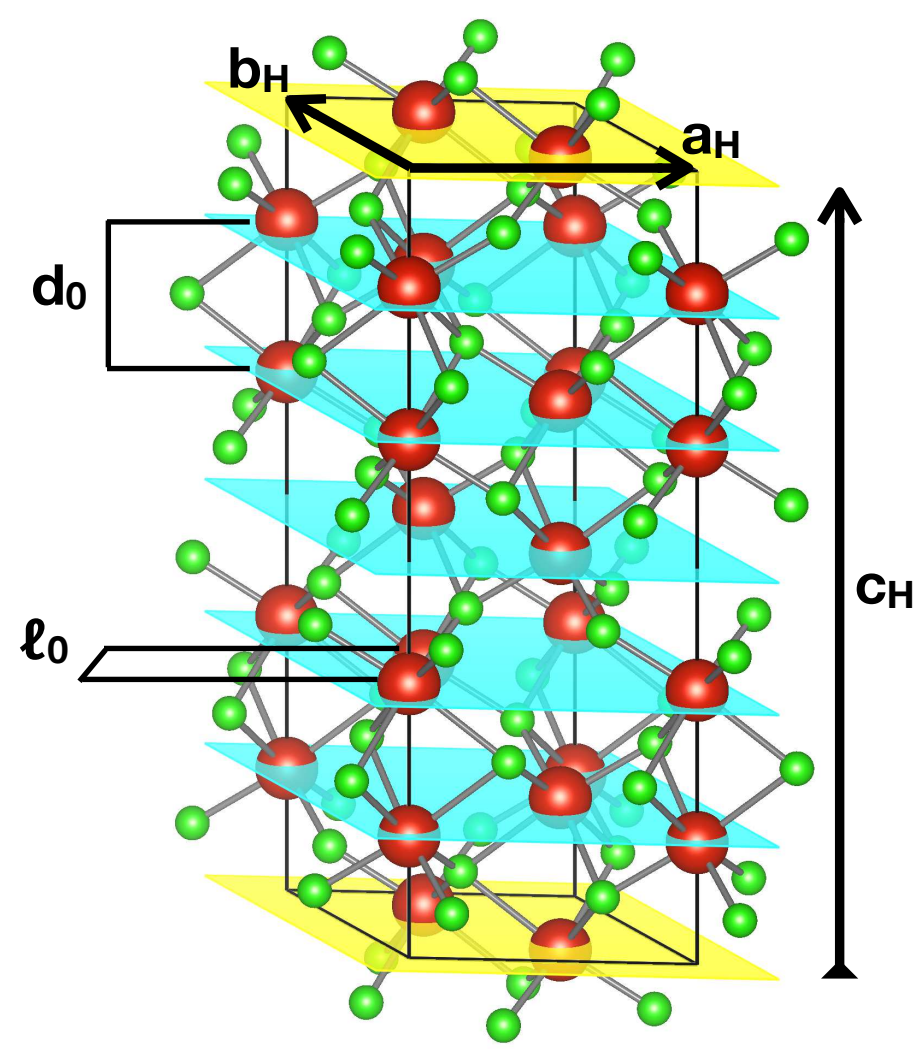

Figure 2: Non-primitive hexagonal unit cell of the high temperature corundum phase. Vanadium is shown in red, while oxygen in green. Note the alternating displacement of the $\mathrm{V}$ atoms off the honeycomb planes. The V-V nearest neighbour distance within the $a_{H}-b_{H}$ plane, $\ell_{0}$, and along $c_{H}, d_{0}$, are also shown. 


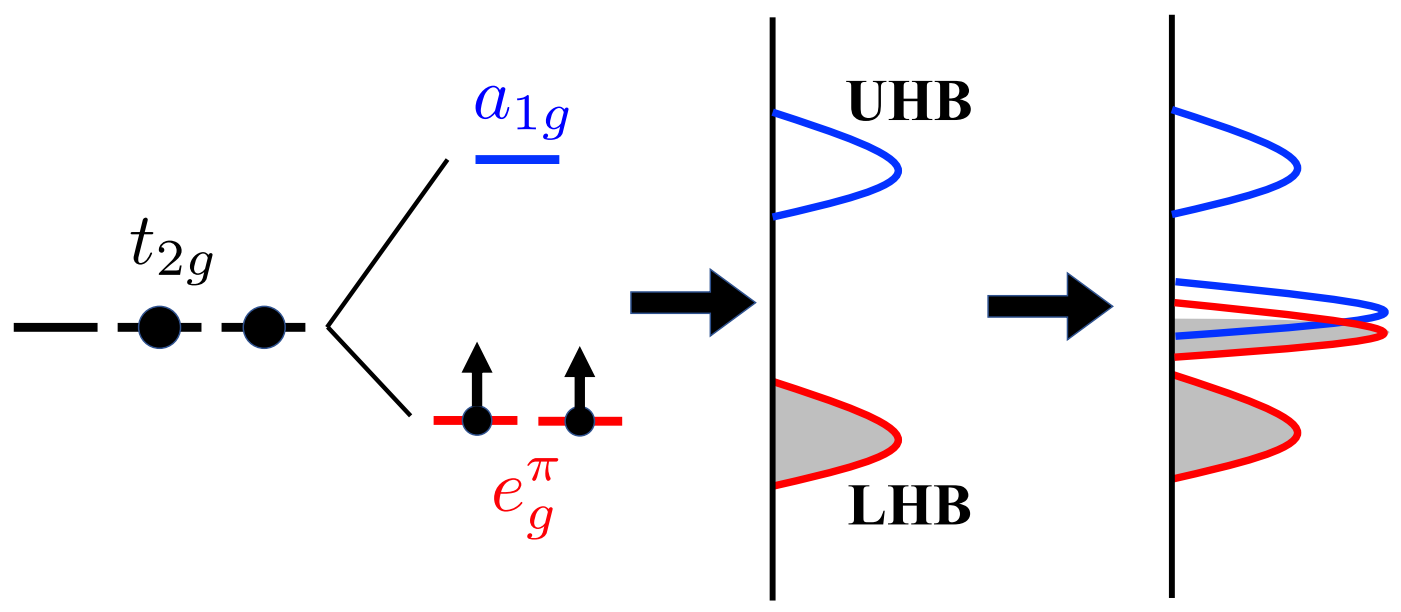

\section{atomic limit $\rightarrow$ Mott insulator $\rightarrow$ metal}

Figure 3: Sketch of the electronic Mott transition in $\mathrm{V}_{2} \mathrm{O}_{3}$. In the atomic limit, the two conduction electrons of $\mathrm{V}^{3+}$ occupy the $t_{2 g}$ orbital of the cubic-field split $3 d$-shell. Because of the additional trigonal field, the $t_{2 g}$ triplet is further split into a lower $e_{g}^{\pi}$ doublet and higher $a_{1 g}$ singlet. The two electrons thus sit into the $e_{g}^{\pi}$ orbital, in a spin triplet configuration because of Hund's rules. In the solid state, this picture remains to a great extent unaltered inside the antiferromagnetic Mott insulator: the atomic levels broaden into lower and upper Hubbard bands, LHB and UHB, respectively. The spin-polarised LHB has prevailing $e_{g}^{\pi}$ character (actually, the $e_{g}^{\pi}$ : $a_{1 g}$ population ratio is 2:1 ${ }^{76}$ ), while the empty UHB has dominant $a_{1 g}$ character. In the metal phase, quasiparticle bands appear at the Fermi level. Since the trigonal field weakens ${ }^{44}$, these bands overlap, thus a metallic behaviour with the simultaneous disappearance of magnetism. 

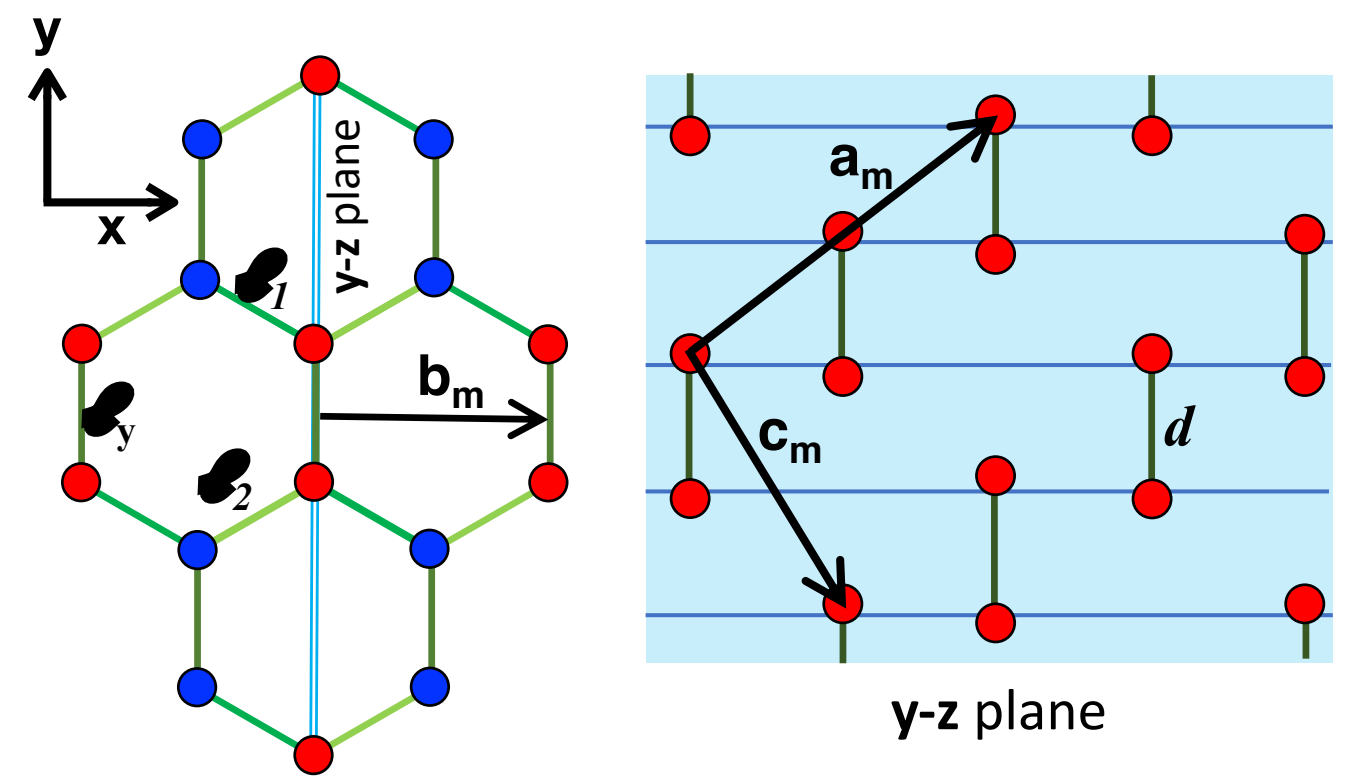

Figure 4: Magnetic order in the hexagonal plane, left panel, and in the $\boldsymbol{y}-\boldsymbol{z}$ plane, equivalent to the $\boldsymbol{a}_{m}-\boldsymbol{c}_{m}$ one, right panel. Red and blue circles correspond to $\mathrm{V}$ atoms with opposite spin. In the hexagonal plane we also draw the nearest neighbour bond lengths: $l_{y}$, which is ferromagnetic, and $l_{1}$ and $l_{2}$, both antiferromagnetic. The dimers, with bond length $d$, lie in the $\boldsymbol{y}-\boldsymbol{z}$ plane and are ferromagnetic, see left panel, where we also show the monoclinic lattice vectors $\boldsymbol{a}_{m}$ and $\boldsymbol{c}_{m}$. 


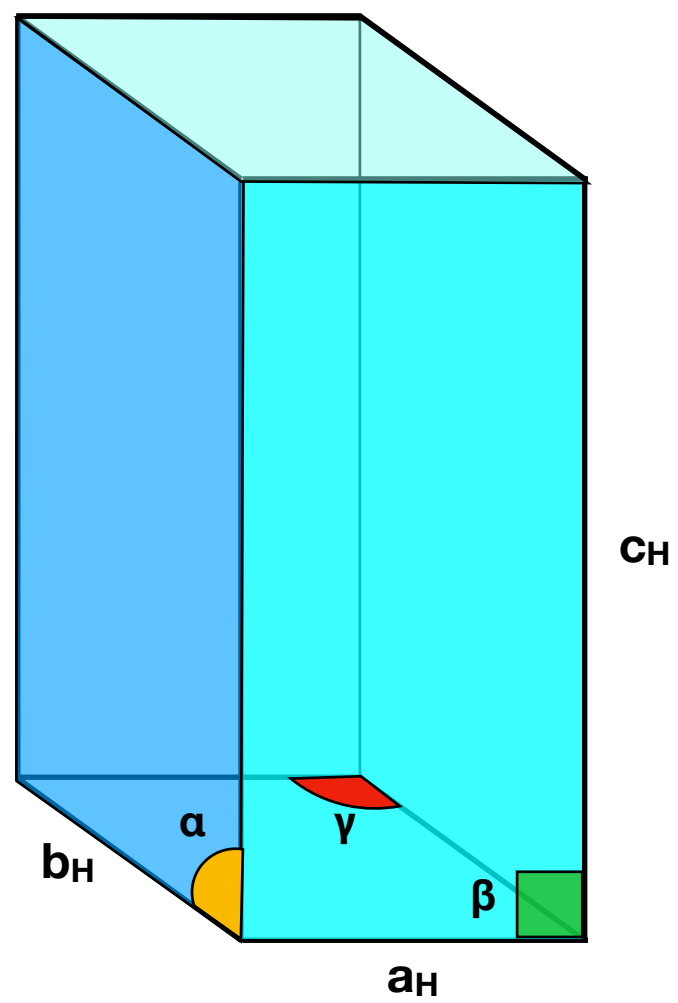

Figure 5: Pseudo-hexagonal unit cell that we use throughout this work. 

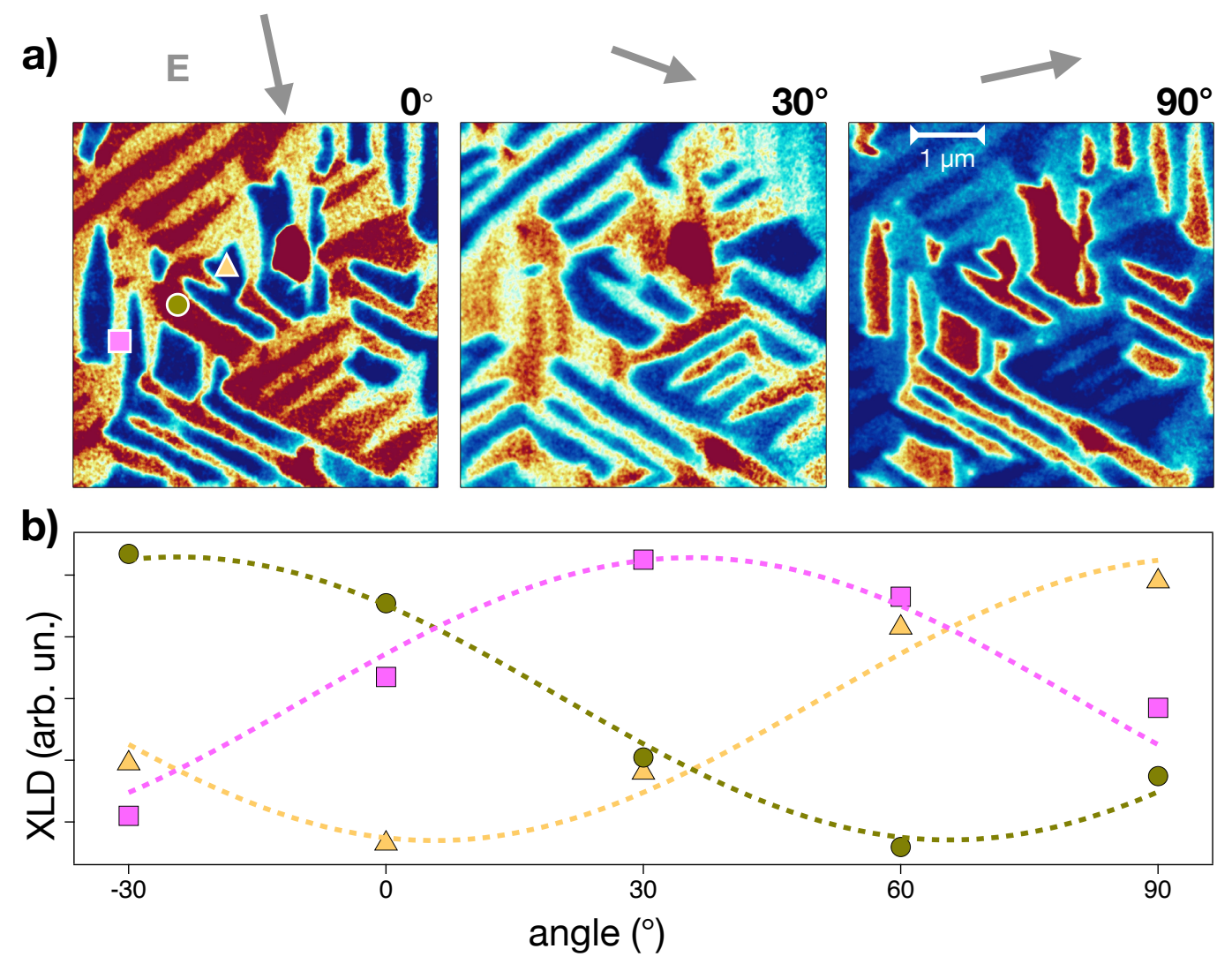

Figure 6: a) XLD-PEEM images taken at $100 \mathrm{~K}$ evidencing striped monoclinic domains characterized by different XLD intensity. The three distinct domains (red, blue, yellow), reflect the three possible monoclinic distortions along the equivalent edges of the vanadium hexagons within the honeycomb planes. The XLD signal, as demonstrated in the Supplementary Section S5 ${ }^{43}$, crucially depends on the orientation between the electromagnetic polarization (grey arrows on top of the images) and the direction of the monoclinic distortion. The three panels show images taken for different polarization angles of the impinging X-ray pulses. The angle indicated refers to the initial (left panel) polarization, which is taken as reference. Note that in the left panel a small angle between the polarization direction and the $y$-axis, as defined in Fig. 4, is present in order to better highlight the three distinct domains. b) XLD signal as a function of the angle between the X-ray polarization and the sample axis. The pink square, green circle and yellow triangle refer to the positions indicated in panel a). Within each domain, the XLD signal displays the expected $180^{\circ}$ periodicity. When comparing the three distinct domains, the XLD signal shows a $60^{\circ}$ phase shift. 


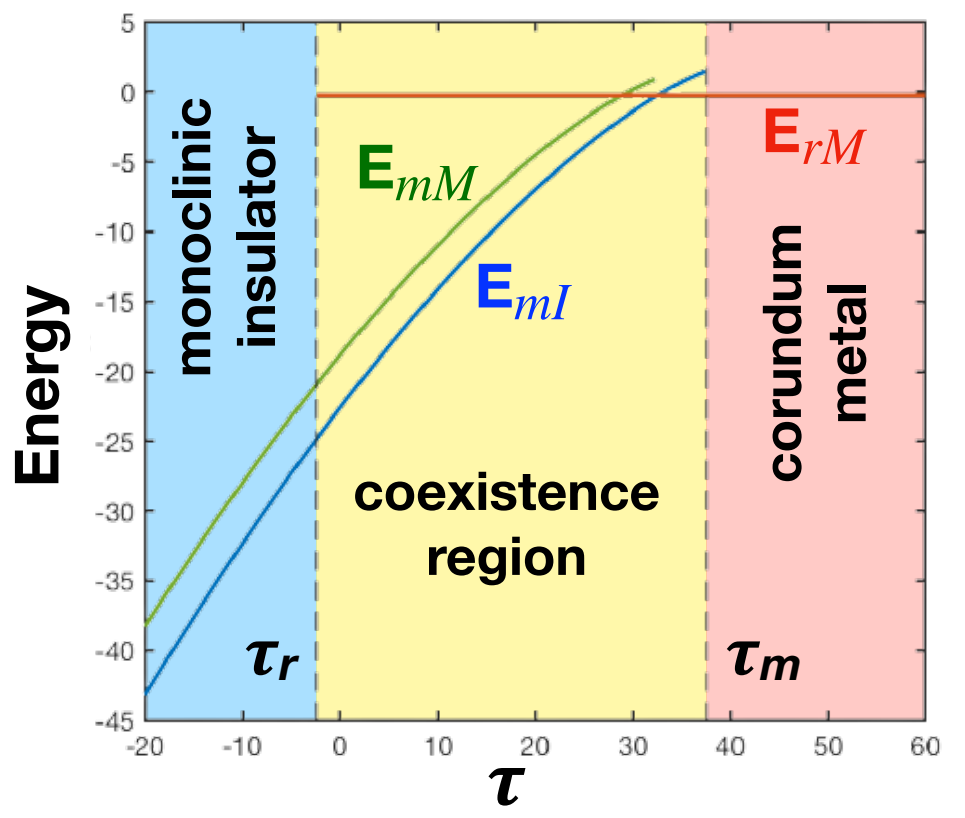

Figure 7: Phase diagram of the energy functional (14) as function of $\tau$ assuming homogeneous phases. $E_{r M}, E_{m M}$ and $E_{m I}$ are, respectively, the energies of the rhombohedral metal, red line, monoclinic metal, green line, and monoclinic insulator, blue line. The energy crossing between $E_{r M}$ and $E_{m I}$ signals the actual first order transition. The vertical dashed lines at $\tau=\tau_{m}$ and $\tau=\tau_{r}$ are, respectively, the monoclinic and rhombohedral spinodal points. For $\tau \in\left[\tau_{r}, \tau_{m}\right]$ there is phase coexistence. We note the existence of a metastable monoclinic metal, with energy $E_{m M}$. 


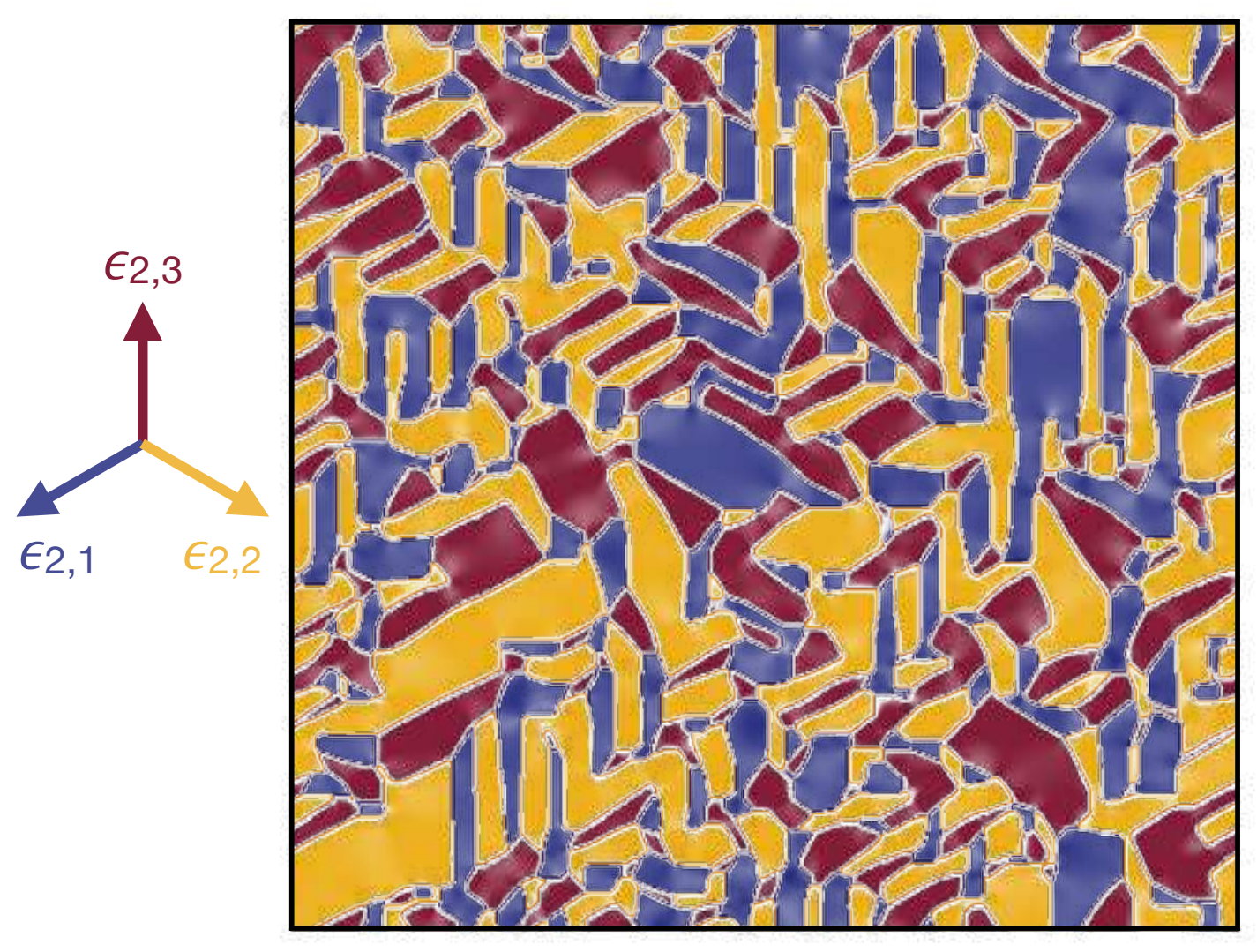

Figure 8: Calculated real space distribution of the shear strain $\epsilon_{2}(\mathbf{r})$ deep inside the monoclinic insulating phase. The colours correspond to the three equivalent shear strain vectors $\boldsymbol{\epsilon}_{2, i}, i=1,2,3$, shown on the left, which characterise the three equivalent monoclinic twins, see Eq. (7). For each domain, the intensity of the color is proportional to the strain amplitude. Lighter regions indicate a reduced strain amplitude. The interfaces between different domains evidently satisfy the curl-free condition (12). 

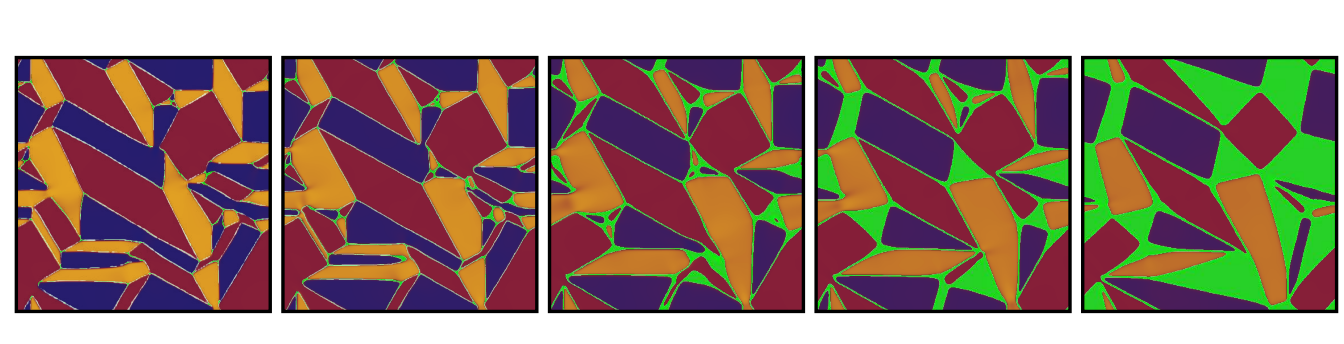

corundum

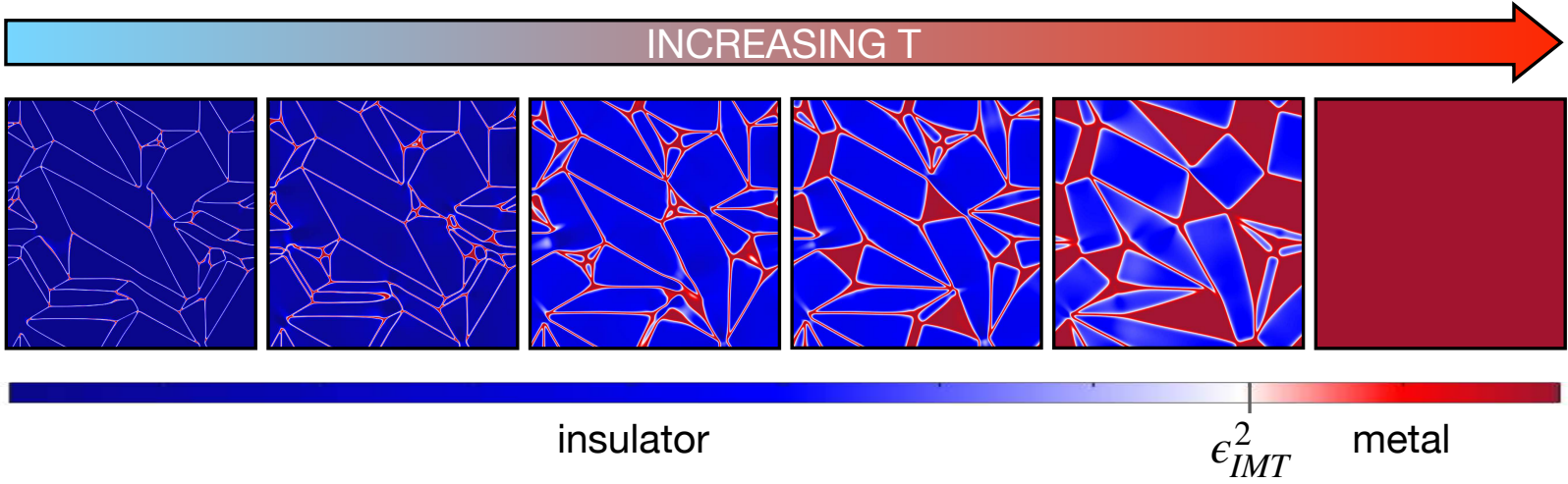

Figure 9: Calculated domain pattern across the first order transition upon rising temperature. Top panel: colour map of the shear strain $\epsilon_{2}(\mathbf{r})$, specifically, the monoclinic domains are indicated by the same colorscale (red, blue, yellow) as that used in Fig. 8. The rhombohedral domains are indicated in green. Bottom panel: colour map of the square modulus of the shear strain $\epsilon_{2}(\mathbf{r})^{2}$. Blue keys indicate the monoclinic insulating domains, $\epsilon_{2}(\mathbf{r})^{2}>\epsilon_{I M T}^{2}$, while red keys the metallic ones, $\epsilon_{2}(\mathbf{r})^{2}<\epsilon_{I M T}^{2}$. 

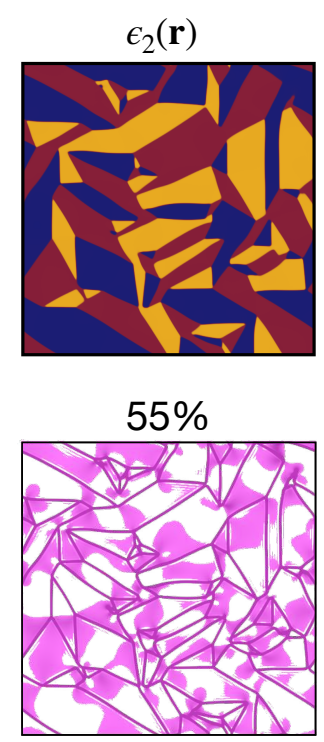

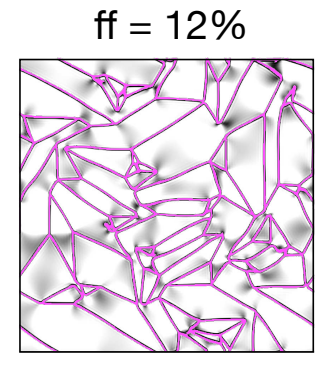

$69 \%$

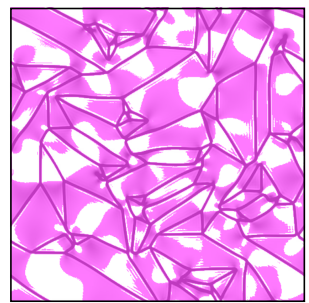

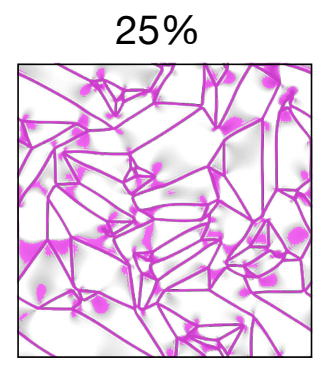

$82 \%$

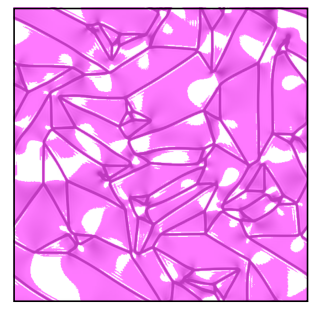

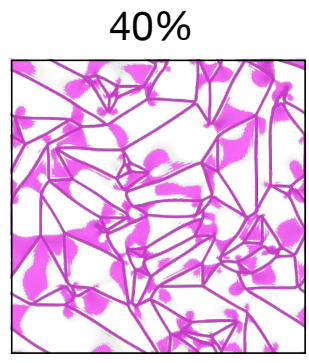

$100 \%$

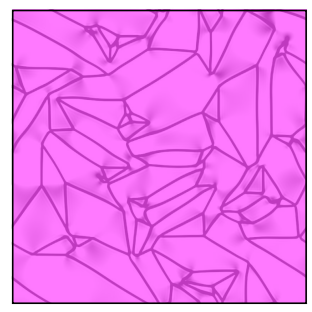

Figure 10: Map of the space-dependent calculated shear strain amplitude (grey color-scale) and metastable monoclinic metallic regions (purple). Darker grey indicates smaller strain amplitude. The purple areas highlight the spatial regions in which the electronic metallic solution with monoclinic strain is the stable one (absolute minimum in the free-energy), i.e. when the condition $\epsilon_{2}(\mathbf{r}) \leq \epsilon_{I M T}(f)$ is fulfilled. We stress that in all panels the same monoclinic shear strain, $\epsilon_{2}(\mathbf{r})$, as that shown in top-left panel (same color scale as Fig. 8) is retained, although not shown for clarity. The filling fraction of the photo-induced non-thermal metallic phase is indicated for each panel. 


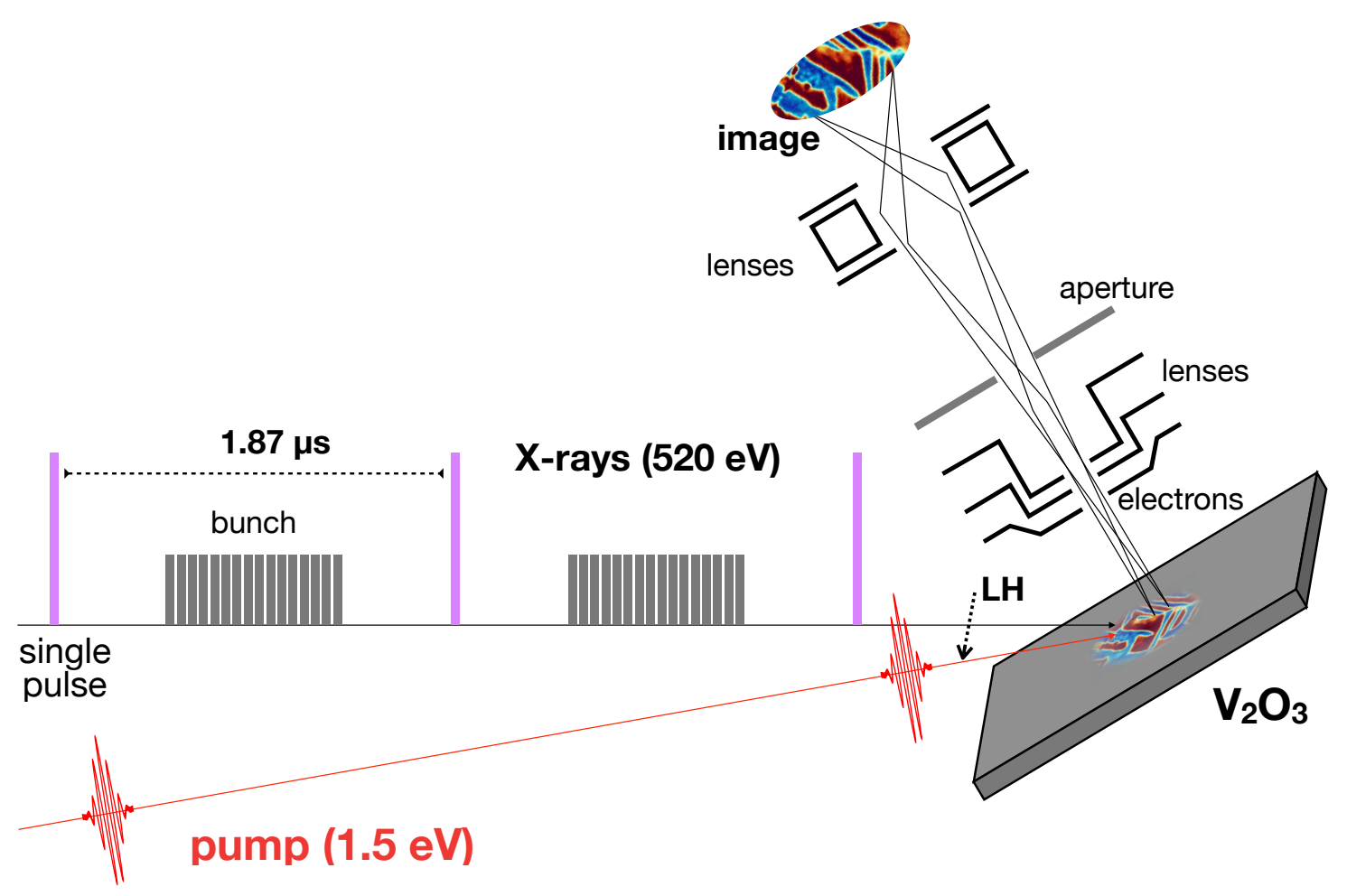

Figure 11: Cartoon of the time-resolved PEEM experiment. The signal originated by isolated Xrays pulses with linear horizontal $(\mathrm{LH})$ polarization is collected by suitable synchronized gating of the X-ray detector. In order to optimize the signal and remove possible artifacts, each reported image is the difference between images taken with X-ray pulses at $520 \mathrm{eV}$, for which the contrast between signals from different monoclinic distortions is maximum, and $518 \mathrm{eV}$, for which the contrast is minimum ${ }^{37,76}$. The pump infrared laser is synchronized to the synchrotron pulses. The electrostatic lenses image the electrons photoemitted from the sample onto the detector. 


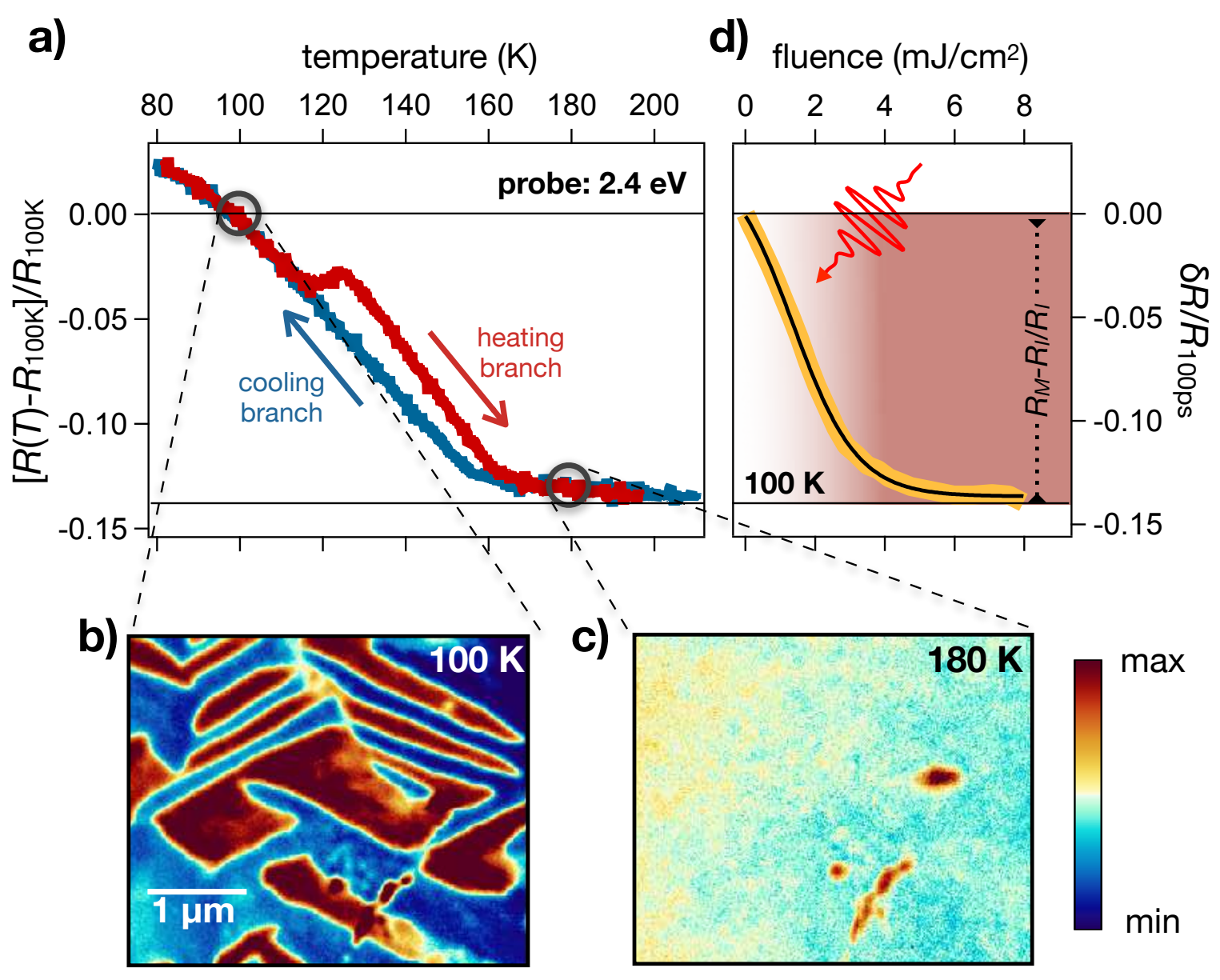

Figure 12: a) Reflectivity change of the $\mathrm{V}_{2} \mathrm{O}_{3}$ crystal across the temperature-driven insulator-tometal phase transition. The sample reflectivity is measured at $2.4 \mathrm{eV}$ photon energy as a function of the sample temperature during the heating (red curve) and cooling (blue curve) processes. The graph displays the relative reflectivity variation with respect to the reflectivity measured at $T=100$ $\mathrm{K}$. b) PEEM image taken at $100 \mathrm{~K}$ evidencing stripe-like domains corresponding to the different monoclinic distortions. Note that the experimental configuration of the image shown (polarization parallel to one of the hexagon edges) is such that only two domains are visible. c) PEEM image taken at $180 \mathrm{~K}$ evidencing a homogeneous background, typical of the metallic corundum phase. The color scale indicates the amplitude of the PEEM signal. d) The asymptotic value of the relative reflectivity variation (yellow trace), i.e. $\delta R / R(100 \mathrm{ps})=[R(\Delta t=100 \mathrm{ps})-R(\Delta t=0 \mathrm{ps})] / R(\Delta t=0 \mathrm{ps})$ where $\Delta t$ is the pump-probe delay, is measured at $2.4 \mathrm{eV}$ probe photon energy and $T=100 \mathrm{~K}$ as a function of the incident pump fluence. The black solid line is a guide to the eye. 


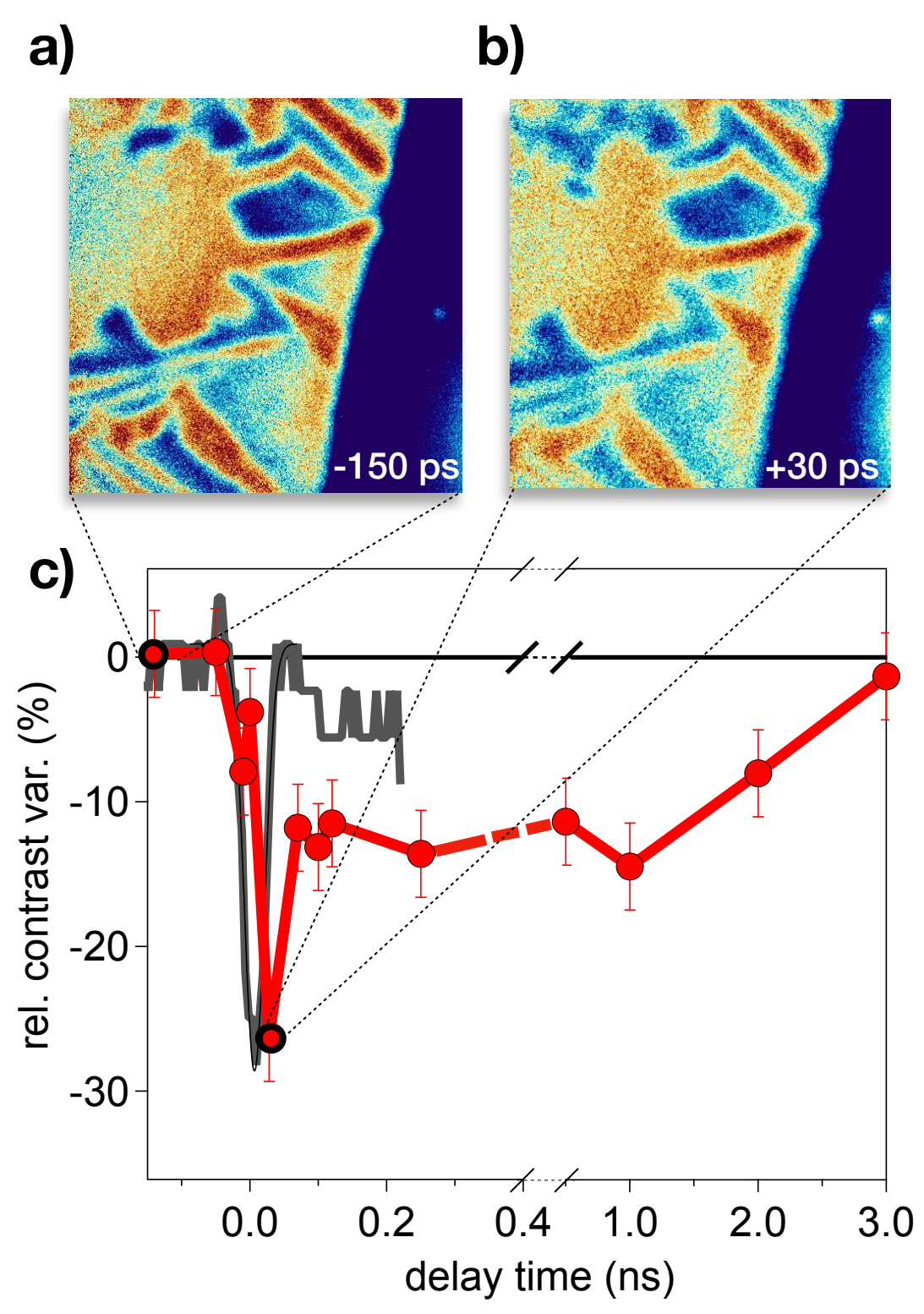

Figure 13: a) Time-resolved PEEM image taken at $T=100 \mathrm{~K}$ and at negative delay (-150 ps) between the infrared pump and the X-ray probe pulses. b) Time-resolved PEEM image taken at $T=100 \mathrm{~K}$ and at positive delay $(+30 \mathrm{ps})$ between the infrared pump and the X-ray probe pulses. The color scale for both panels a) and b) is the same than that used in Fig. 12. c) Relative contrast (see the Supplemental Material ${ }^{43}$ ) between different domains as a function of the delay between the infrared pump and the X-ray probe pulses. The error bar accounts for the average fluctuation of the signal within the domains considered for the calculation of the relative contrast. The grey solid line is the cross correlation between the infrared pump and the X-ray probe pulses measured by exploiting the non-linear photoemission from surface impurities on the sample (see the Supplementary Material ${ }^{43}$ ). 

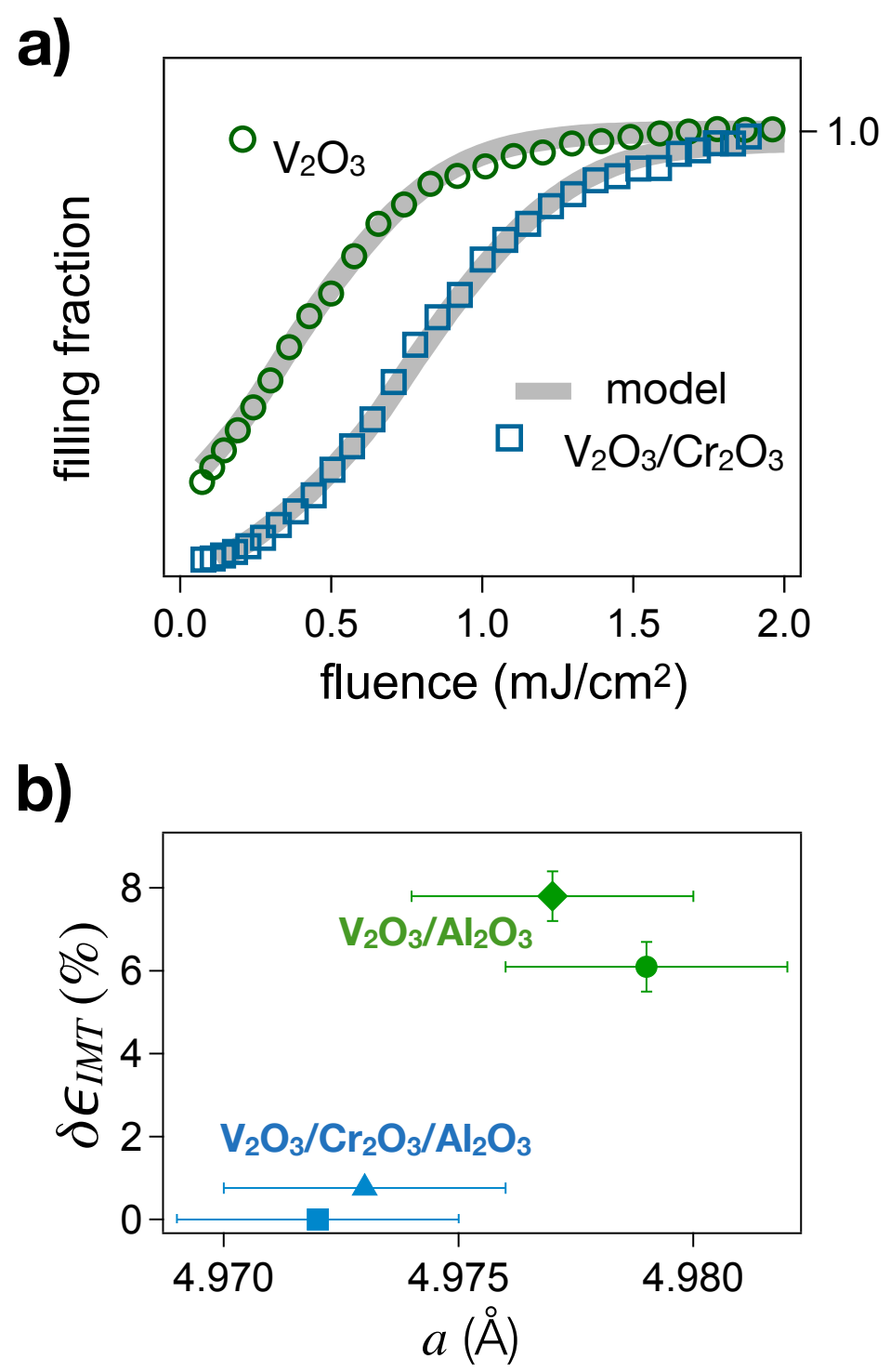

Figure 14: a) Metallic filling fraction, retrieved from the asymptotic value of the relative reflectivity variation, i.e. $\delta R / R(100 \mathrm{ps})$, as a function of the pump incident fluence for a $50 \mathrm{~nm} \mathrm{~V}_{2} \mathrm{O}_{3}$ film directly grown on the sapphire substrate (green circles) and for a $55 \mathrm{~nm} V_{2} \mathrm{O}_{3}$ film grown on a $60 \mathrm{~nm} \mathrm{Cr} \mathrm{O}_{3}$ buffer layer (blue squares). The grey solid lines represent the numerical filling fractions, calculated as the ratio between non-thermal metallic areas (purple areas in Fig. 10)) and the total area. b) Values of the estimated critical strain variation $\delta \epsilon_{I M T}$, calculated with respect to the reference sample $\mathrm{V}_{2} \mathrm{O}_{3} / \mathrm{Cr}_{2} \mathrm{O}_{3} / \mathrm{Al}_{2} \mathrm{O}_{3}(55 \mathrm{~nm} / 60 \mathrm{~nm} /$ substrate $) . \delta \epsilon_{I M T}$ is plotted as a function of the room temperature $a$-axis lattice parameter, as measured by X-ray diffraction, for samples with (blue points) and without (green points) the $\mathrm{Cr}_{2} \mathrm{O}_{3}$ buffer layer. The symbols refer to the following samples: blue square $\mathrm{V}_{2} \mathrm{O}_{3} / \mathrm{Cr}_{2} \mathrm{O}_{3} / \mathrm{Al}_{2} \mathrm{O}_{3}(55 \mathrm{~nm} / 60 \mathrm{~nm} / \mathrm{substrate})$; blue triangle $\mathrm{V}_{2} \mathrm{O}_{3} / \mathrm{Cr}_{2} \mathrm{O}_{3} / \mathrm{Al}_{2} \mathrm{O}_{3}(67 \mathrm{~nm} / 40 \mathrm{~nm} /$ substrate $)$; green diamond $\mathrm{V}_{2} \mathrm{O}_{3} / \mathrm{Al}_{2} \mathrm{O}_{3}(40 \mathrm{~nm} /$ substrate); green circle $\mathrm{V}_{2} \mathrm{O}_{3} / \mathrm{Al}_{2} \mathrm{O}_{3}(50 \mathrm{~nm} /$ substrate $)$. 


\section{Supplementary Files}

This is a list of supplementary files associated with this preprint. Click to download.

- supplementary.pdf 